\title{
Alterations in Sucrose and Phenylpropanoid Metabolism Affected by BABA-Primed Defense in Postharvest Grapes and the Associated Transcriptional Mechanism
}

\author{
Chunhong Li, ${ }^{1,2}$ Kaituo Wang, ${ }^{1, \dagger}$ Changyi Lei, ${ }^{1}$ Shifeng Cao, ${ }^{3}$ Yixiao Huang, ${ }^{1,4}$ Nana $\mathrm{Ji}^{2}{ }^{2}$ Feng $\mathrm{Xu}^{2}$ and \\ Yonghua Zheng ${ }^{2}$ \\ ${ }^{1}$ College of Biology and Food Engineering, Chongqing Three Gorges University, Chongqing 404000, China \\ ${ }^{2}$ College of Food Science and Technology, Nanjing Agricultural University, Nanjing 210095 Jiangsu, China \\ ${ }^{3}$ College of Biological and Environmental Sciences, Zhejiang Wanli University, Ningbo 315211, China \\ ${ }^{4}$ College of Arts and Sciences, University of Miami, Coral Gables, FL 33143, U.S.A.
}

Accepted 19 July 2021.

Defense elicitors can induce fruit disease resistance to control postharvest decay but may incur quality impairment. Our present work aimed to investigate the resistance against Botrytis cinerea induced by the elicitor $\beta$-aminobutyric acid (BABA) and to elucidate the specific transcriptional mechanism implicated in defense-related metabolic regulations. The functional dissection results demonstrated that, after inoculation with the fungal necrotroph $B$. cinerea, a suite of critical genes encoding enzymes related to the sucrose metabolism and phenylpropanoid pathway in priming defense in grapes were transcriptionally induced by treatment with $10 \mathrm{mM}$ BABA. In contrast, more UDP-glucose, a shared precursor of phenylpropanoid and sucrose metabolism, may be redirected to the phenylpropanoid pathway for the synthesis of phytoalexins, including trans-resveratrol and $\varepsilon$-viniferin, in $100 \mathrm{mM}$ BABA-treated grapes, resulting in direct resistance but compromised soluble sugar contents. An R2R3-type MYB protein from Vitis vinifera, VvMYB44, was isolated and characterized. $V v M Y B 44$ expression was significantly induced upon the grapes expressed defensive reaction. Subcellular localization, yeast two-hybrid, and coimmunoprecipitation assays revealed that the nuclear-localized $V v M Y B 44$ physically interacted with the salicylic acid-responsive transcription coactivator NPR1 in vivo for defense expression. In addition, VvMYB44 directly bound to the promoter regions of sucrose and phenylpropanoid metabolism-related genes and transactivated their expression, thus tipping the balance of antifungal compound accumulation and soluble sugar maintenance. Hence, these results suggest that 2R-type $V v M Y B 44$ might be a potential positive participant in BABA-induced priming

${ }^{\dagger}$ Corresponding author: K. Wang; wangkaituo83@gmail.com

Funding: This study was supported by the National Natural Science Foundation of China (number 31671913), the Natural Science Foundation of Ningbo City (number 2018A610224) and the Open Foundation for Institute of Three Gorges research (number 2019sxxyjd08).

*The $\boldsymbol{e}$-Xtra logo stands for "electronic extra" and indicates there are supplementary materials published online.

The author(s) declare no conflict of interest.

(c) (1) () $\odot$ Copyright $(02021$ The Author(s). This is an open access article (c) 1 . No distributed under the CC BY-NC-ND 4.0 International license. defense in grape berries that contributes to avoiding the excessive consumption of soluble sugars during the postharvest storage.

Keywords: $\beta$-aminobutyric acid, Botrytis cinerea, defense response, grape berries, MYB transcription factor, quality maintenance

Grapevine (Vitis vinifera L.) is a substantial important horticultural crop worldwide due to the pleasant aromatic flavor and plentiful phytonutrient profiles of its fruit tissues (Dai et al. 2011). Nevertheless, grape berry is extremely susceptible to infection by microbial phytopathogens, leading to enormous wastage if the protective management is not utilized. Gray mold disease caused by the necrotrophic fungus Botrytis cinerea jeopardizes the survival of many plant species and is a severe disease in grape berries (Feliziani et al. 2013; Zhang et al. 2014). Persistent fungicides sprayed on grapevine were effective in the control of gray mold infection, while public concern regarding residual chemicals in the environment has limited the application of fungicides (Schirra et al. 2011). Eco-compatible alternatives to chemical fungicides for the inhibition of gray mold rot in grapes are pressingly demanded. Among recently applied sustainable strategies, the induction of broad-spectrum defense resistance in plants can be achieved by several natural elicitors, among which the nonproteinogenic amino acid $\beta$-aminobutyric acid (BABA) has been reported as a promising elicitor involved in activating defense responses in several horticultural crops (Cohen et al. 2016; Mostek et al. 2016; Wilkinson et al. 2018). BABA-induced resistance (BABA-IR) depends not only on salicylic acid (SA) signaling transduction but also on the perception of specific abscisic acid (ABA)- or phosphoinositide-dependent signaling (Ton and Mauch-Mani 2004; Luna et al., 2014; Ton et al. 2005; Zimmerli et al. 2000). Specifically, BABA-IR occurs in two different manners, mainly depending on dose (Zimmerli et al. 2001). Direct defense confers simultaneous resistance following a relatively high concentration of BABA elicitation (100 to $500 \mathrm{mM}$ ) (Wang et al. 2016), whereas priming defense can prepare the defense system to mount a comparatively rapid and robust response, following treatment with a suitable BABA concentration (10 to $50 \mathrm{mM}$ ), when the fruit is subjected to pathogen invasion (Conrath et al. 2006; Jung et al. 2009; Li et al. 2020; Wang et al. 2019). Priming resistance is a critical regulatory system in which the defense arsenal in alarmed or primed plants remains dormant until they are exposed to disease stress. 
Hence, priming resistance represents a more efficient mode of IR, since the priming defense process can balance disease protection and fitness costs (van Hulten et al. 2006). Our previous research also demonstrated that $\mathrm{BABA}$-activated priming defense contributes to postharvest decay control through the priming of pathogen-dependent resistance, particularly by potentiating the gene expression of SA-responsive pathogenesisrelated $(P R) P R 1, P R 2$, and $P R 5$ genes and maintaining fruit quality through soluble sugar accumulation, while direct defense imposes redundant metabolic costs and negatively affects soluble sugar concentrations in grape berries and peach fruit ( $\mathrm{Li}$ et al. 2021a and b; Wang et al. 2015, 2016). Metabolizable sugars not only play nutritive roles in fruit sensory characteristics but also exert regulatory functions as signaling molecules in defense responses (Borsani et al. 2009; Morkunas and Ratajczak 2014; Qin et al. 2016). In the sucrose metabolic system, UDP-glucose (UDPG) is recognized as the central precursor in the pathways of both sucrose synthesis and degradation (Ciereszko et al. 2001). Our previous publication on elicitor IR in grape cells revealed that the increased amount of UDPG derived from hydrolyzed sucrose could be directed to the synthesis of stilbene phytoalexins through the phenylpropanoid pathway as a defensive response to fungal invasion (Wang et al. 2015), demonstrating a close affiliation between sugar metabolism and the phenylpropanoid pathway in plant disease resistance. Hence, the BABA-IR mode linked with the direction and strength of secondary metabolism adds another dimension to the complexities of plant defense responses.

Similar to plant immune responses such as pattern-triggered immunity and effector-triggered immunity and many other complex characteristics of IR, including systemic acquired resistance (SAR), induced systemic resistance, and volatile organic compound IR, BABA-IR requires the large-scale transcriptional reprogramming of plant host genes encoding regulatory and antimicrobial proteins regulated by the specific recognition of transcription factors (TFs) (de Leone et al. 2020; Hill and Treisman 1995; Vaahtera and Brosché 2011). The involvement of some classes of plant-specific TFs has been implicated in pathogenstimulated responses, including DNA-binding proteins harboring a highly conserved MYB domain of 50 to 53 amino acid residues (Dubos et al. 2010; Katiyar et al. 2012; Meissner et al. 1999; Peng et al. 2016). Most MYB TFs bind to one or more MYB-binding sites (MBSs) of two different types, consisting of the consensus sequences of MBS I [CNGTT(A/G)] and MBS II $[\mathrm{C}(\mathrm{G} / \mathrm{T}) \mathrm{T}(\mathrm{A} / \mathrm{T}) \mathrm{GTT}(\mathrm{A} / \mathrm{G})]$, within their downstream target genes (Romero et al. 1998). According to the number of adjacent repeats that form helix-turn-helix (HTH) conformations, MYB TFs are grouped into four subfamilies, comprising 1R-MYB (with one repeat), 2R-MYB (R2R3-MYB, with two repeats), 3R-MYB (R1R2R3-MYB, with three repeats), and 4R-MYB (R0R1R2R3-MYB, with four repeats) (Du et al. 2013; Dubos et al. 2010). Among MYBs in plants, numerous R2R3-type MYBs (the largest subfamily) have been characterized partly through genetic approaches (Martin and Paz-Ares 1997; Stracke et al. 2001) and have been shown to be involved in the regulation of many plant-specific metabolic processes (Gonzalez et al. 2008; Stracke et al. 2007; Zhong et al. 2007; Zhou et al. 2009) and the regulation of organ development in Arabidopsis (Cheng et al. 2009; Zhang et al. 2007). In addition, some R2R3-type MYBs have essential functions in defense reaction against fungal pathogens in the plant pathosystem. AtMYB108 regulates Arabidopsis resistance against the necrotrophic fungi $B$. cinerea and Alternaria brassicicola (Mengiste et al. 2003). The overexpression of AtMYB30 intensifies the resistance of transgenic Arabidopsis and tobacco to avirulent and virulent bacterial and fungal pathogens (Vailleau et al. 2002). Arabidopsis overexpressing MYB96 shows potentiated resistance to Pseudomona syringae pv. tomato DC3000 (Seo and Park 2010). Moreover, AtMYB15 is a positive regulator of basal immunity, and the myb15 mutant exhibits accelerated susceptibility to the foliar bacterial pathogen $P$. syringae (Chezem et al. 2017). Recently, the roles of another R2R3-MYB protein, MYB44, have been well-characterized not only in insect resistance (Liu et al. 2010; Lü et al. 2011) and stress tolerance (Jung et al. 2008; Persak and Pitzschke 2014; Seo et al. 2012) but also in fungal pathogen defense (Hieno et al. 2016; Qiu et al. 2019; Zou et al. 2013) in both Arabidopsis and eggplant, and the overexpression of AtMYB44 can evidently upregulate the transcript levels of WRKY70 (an important regulator within the SA signaling pathway) and PRI (an SA-responsive gene) in transgenic Arabidopsis (Shim et al. 2013; Zou et al. 2013). Nevertheless, the role of $V$. vinifera MYBs $(V v M Y B s)$ in the transcriptional manipulation of metabolic processes, particularly the phenylpropanoid pathway and sugar metabolism, and the molecular mechanism underlying MYB-derived BABA-IR in the response of grape berries to fungal pathogen attack are still unknown. In the current study, we adopted high-throughput Illumina RNA sequencing (RNA-seq) to analyze the transcriptional responses in BABA-elicited defense against $B$. cinerea and further dissected the potential MYB TF-mediated transcriptional regulation of BABA-induced alterations in the secondary metabolism of grape berries related to the specific resistance mode induced by different BABA concentrations.

\section{RESULTS}

Effect of BABA treatment on the inhibition of $B$. cinerea infection and physiological responses in grape berries.

Grapes are susceptible to gray mold caused by the fungus B. cinerea (Fig. 1A and B), and the artificial spraying of berries with $B$. cinerea suspensions gives rise to mold spots on the surface of their pericarps. Notably, BABA treatment at 10 or $100 \mathrm{mM}$ exerted a significant $(P<0.05)$ reduction in decay incidence when compared with that in the control berries over the time course of incubation at $20^{\circ} \mathrm{C}$. Interestingly, BABA treatment at a concentration of 10 or $100 \mathrm{mM}$ caused no significant $(P>0.05)$ difference in the efficacy of controlling disease development. In addition, BABA treatments significantly retarded the increase on respiration rate, ethylene (ET) production, and malondialdehyde (MDA) content but promoted an $\mathrm{H}_{2} \mathrm{O}_{2}$ burst in the defense response of grape berries against postharvest fungal invasion compared with the controls throughout the storage. Specially, the promotion effect on the $\mathrm{H}_{2} \mathrm{O}_{2}$ content and the inhibitory effect on the physiological responses of BABA treatment at $10 \mathrm{mM}$ were stronger than at $100 \mathrm{mM}$ BABA (Fig. 1C through F).

\section{Effect of BABA treatment on the contents of soluble sugars, UDPG, and phenylpropanoid metabolites in grape berries.}

In control grapes, the contents of glucose, fructose, and sucrose continuously accumulated in a similar pattern over the incubation at $20^{\circ} \mathrm{C}$ for 5 days. Treatment of grape berries with $10 \mathrm{mM}$ BABA increased the level of soluble sugars, with the sugar contents of grapes treated with $100 \mathrm{mM}$ BABA being slightly lower than the controls (Fig. 2A through C). On the other hand, the UDPG content in the control grapes did not exhibit any statistically significant change throughout the incubation period, while the control berries exhibited a slightly increasing trend of trans-resveratrol content but a stable content of $\varepsilon$-viniferin over the 5-day incubation period. The UDPG content was enhanced by treatment with $\mathrm{BABA}$ at $100 \mathrm{mM}$ relative to that in the control grapes. 
Meanwhile, treatment with 10 or $100 \mathrm{mM}$ BABA led to significant increases in stilbenes, including trans-resveratrol and its oligomer $\varepsilon$-viniferin, and no statistically significant difference was found between them at each timepoint following inoculation at $20^{\circ} \mathrm{C}$ (Fig. 2D through F).
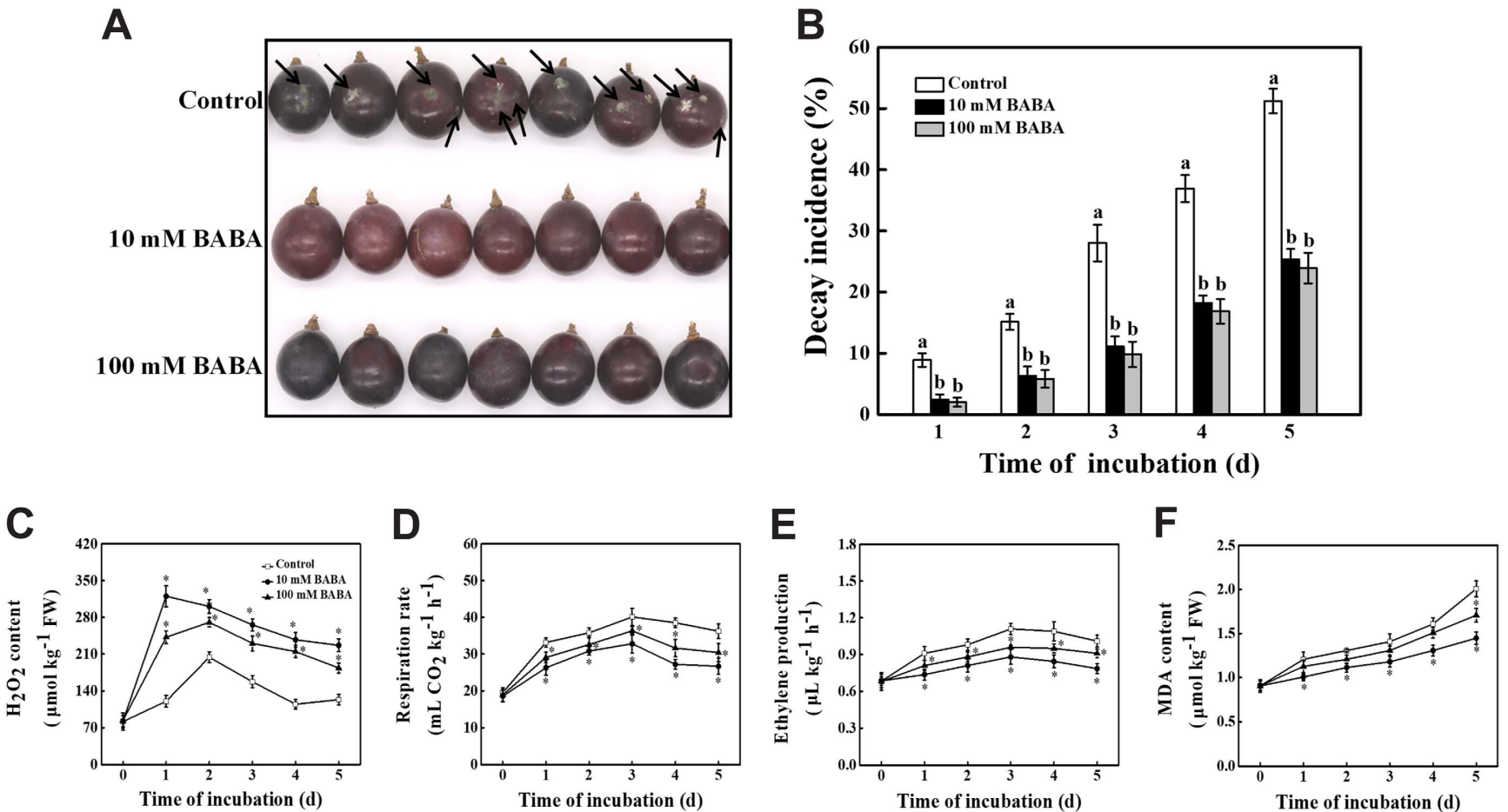

Fig. 1. A, Changes in disease phenotype, B, decay incidence, $\mathbf{C}, \mathrm{H}_{2} \mathrm{O}_{2}$ content, D, respiration rate, E, ethylene production and F, malondialdehyde (MDA) content in grapes under $\beta$-aminobutyric acid (BABA) treatments at 0,10 , and $100 \mathrm{mM}$ during incubation at $20^{\circ} \mathrm{C}$ for 5 days. Data are recorded as the mean \pm standard error (SE) of nine assessments. Statistical vertical bars represent SE. Different letters or asterisk $\left({ }^{*}\right)$ indicate significant $(P<$ $0.05)$ differences between BABA treatments and the control. The BABA treatments experiment was conducted twice with similar results, and the image of postharvest grapes under BABA treatments at different concentrations derive from the second repeat experiment.
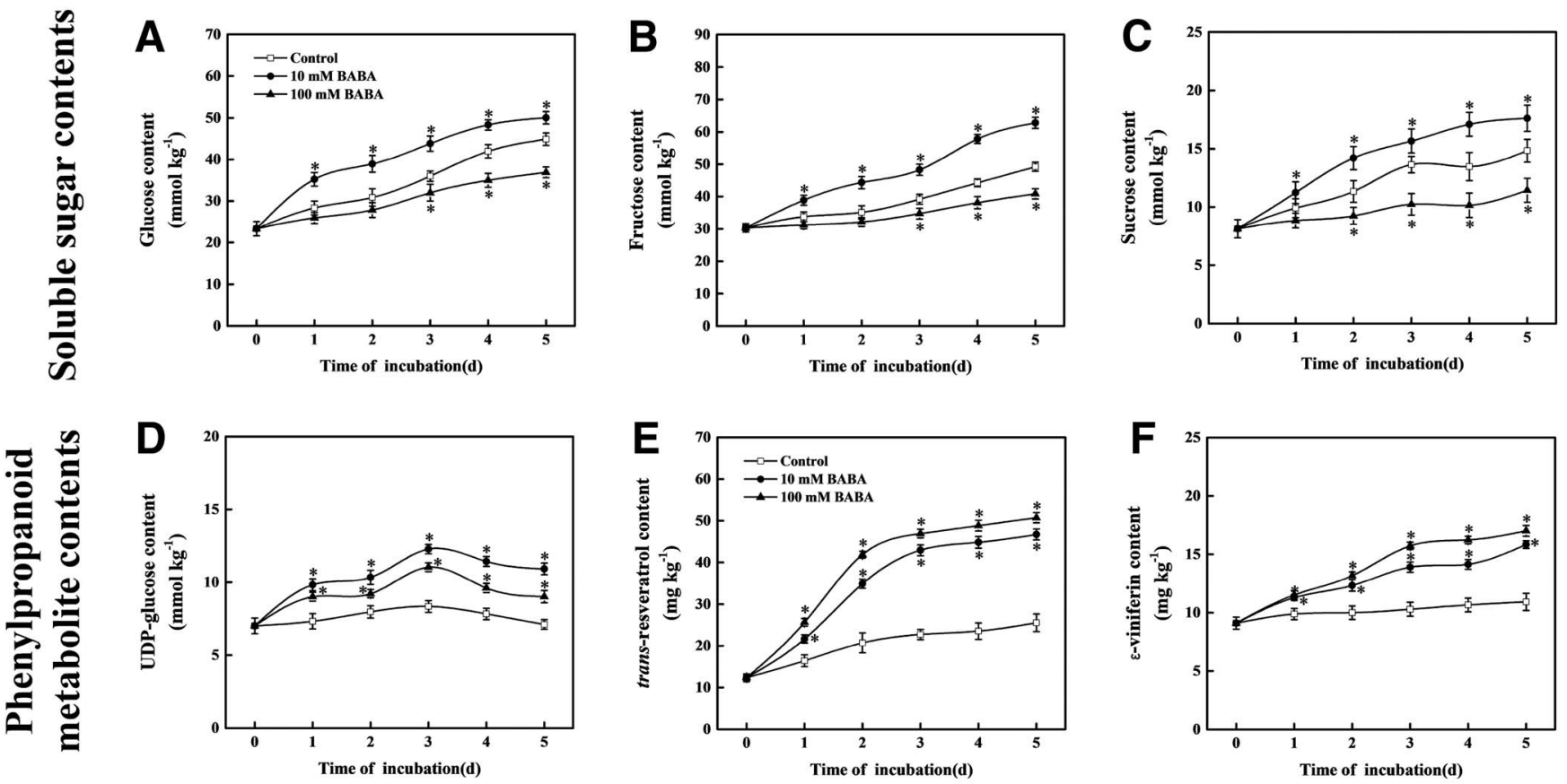

Fig. 2. $\beta$-Aminobutyric acid (BABA) treatments affected the contents of soluble sugars, including $\mathbf{A}$, glucose, $\mathbf{B}$, fructose and $\mathbf{C}$, sucrose, and of phenylpropanoid metabolites, including D, UDP-glucose, E, trans-resveratrol, and $\mathbf{F}, \boldsymbol{\varepsilon}$-viniferin, in grape berries. Data are recorded as the mean \pm standard error (SE) of triplicate assays. Statistical vertical bars represent SE. An asterisk $\left(^{*}\right)$ indicates significant differences between BABA-treated and untreated berries $(P=0.05)$. 
lyases] genes, $V v I C S 2$ [isochorismate synthase 2], $V v C M$ [chorismate mutase] genes, $V v B A 2 H$ [benzoic acid 2-hydroxylase] genes, and $V v I P L$ [isochorismate pyruvate lyase] genes) in the berries treated with 10 or $100 \mathrm{mM}$ BABA followed by B. cinerea inoculation noticeably increased compared with the levels in grapes solely inoculated with $B$. cinerea. In addition, $10 \mathrm{mM}$ BABA-treated berries exhibited a higher SA content and increased SA biosynthesis gene expressions than grapes treated with $100 \mathrm{mM}$ BABA (Fig. 3A and B). Moreover, the combination of BABA treatment with pathogen inoculation enhanced the expression profiles of SA-responsive transcription coactivator VvNPR1 (nonexpressor of pathogen-related gene 1), SA-inducible $V v P R l$ (encoding a basic PR protein 1) and two defense-related enzymes, including $V v G N S$ ( $\beta$-1,3-glucanase) and $V v C H I$ (chitinase) (Fig. 3C). More specifically, the expression levels of $V v N P R 1, V v P R 1, V v G N S$, and $V v C H I$ in the grape berries treated with $10 \mathrm{mM}$ BABA transiently increased to maximum levels on the first day of incubation and then decreased gradually in the remaining days. In contrast, BABA treatment at $100 \mathrm{mM}$ induced sharper and more sustained transcripts of $P R$ genes compared with those observed in the $10 \mathrm{mM}$ BABAtreated fruit. However, the visible accumulation of jasmonate (JA) or ET-dependent genes, such as VvPDF1.2 (encoding a plant defensin) and $\mathrm{VvHel}$ (encoding a hevein-like protein), could not be detected after BABA elicitation (Fig. 3C).

\section{Transcriptional analysis of BABA-IR.}

RNA-seq analysis generated 51,012,266, 53,014,730, and $52,193,566$ clean reads for the control, $10 \mathrm{mM}$ BABA-, and $100 \mathrm{mM}$ BABA-treated grapes, respectively, in which the average proportion was $97.83 \%$. Through transcriptome reconstruction, 18,328, 18,219, and 18,443 unigenes were achieved from $0,10 \mathrm{mM}$ and $100 \mathrm{mM}$ BABA-treated berries, respectively (Supplementary Table S2). These genes were further subjected to filtering of differentially expressed genes (DEGs) corresponding to the pairwise comparisons of $10 \mathrm{mM}$ BABA-vs-control, $100 \mathrm{mM}$ BABA-vs-control, and $10 \mathrm{mM}$ BABA-vs-100 mM BABA by the DESeq R package. A total of 3,040 DEGs were included in a Venn diagram (Fig. 4A), including 1,371 up- and 1,669 downregulated DEGs. Among these DEGs, 871 and 718 were up- and down-regulated, respectively, in $10 \mathrm{mM} \mathrm{BABA-}$ induced grapes, and 656 and 1,344 DEGs were up- and downregulated in $100 \mathrm{mM} \mathrm{BABA}$-induced berries relative to the controls. In addition, 414 up- and 467 downregulated DEGs were shared between the $10 \mathrm{mM}$ BABA-vs-control and $100 \mathrm{mM}$ BABA-vs-control pairwise comparisons. In addition, the treatment of berries with $10 \mathrm{mM}$ BABA primed 308 up- and 102 downregulated DEGs relative to the $B$. cinerea-inoculated fruit subjected to BABA treatment at $100 \mathrm{mM}$. These data illustrated an extremely similar state with slight differences in the transcriptomic responses of grape berries in the IR elicited by different BABA concentrations.

\section{Gene ontology (GO) analysis of DEGs.}

A total of 349 upregulated DEGs identified in the $10 \mathrm{mM}$ BABA-vs-control pairwise comparison were classified into 37 GO categories and annotated with 591 GO terms, among which 161 DEGs were assigned to 16 categories and 440 GO terms in the biological process category, 114 DEGs were assigned to 11 categories and $94 \mathrm{GO}$ subcategories in the cellular component category, and 74 DEGs were assigned to 10 categories and 57 subcategories in the molecular function category (Fig. 4B; Supplementary Table S4). In the $100 \mathrm{mM}$ BABA-vs-control pairwise comparison, 408 downregulated DEGs were classified into 38 GO categories and annotated with 322 terms (Fig. 4C; Supplementary Table S4). Additionally, 106 upregulated DEGs were classified into $28 \mathrm{GO}$ categories and were annotated with
$336 \mathrm{GO}$ terms in the $10 \mathrm{mM}$ BABA-vs-100 mM BABA pairwise comparison, among which 53 DEGs were assigned to 14 categories and $215 \mathrm{GO}$ terms in the biological process category, 29 DEGs were assigned to nine categories and $78 \mathrm{GO}$ terms in the cellular component category, and 24 DEGs were assigned to five categories and $43 \mathrm{GO}$ terms in the molecular function category (Fig. 4D; Supplementary Table S4). In each of the main $\mathrm{GO}$ categories obtained from the three pairwise comparisons of the 'metabolic process' (among biological processes), 'cell' (among cell components), and 'catalytic activity' (among molecular functions) terms were the most highly represented, which illustrated that the numerous metabolic activities of grapes were activated by BABA treatments and that these processes were modulated by various genes within the cells.

Furthermore, GO terms of DEGs assigned to the biological process category showed that the overrepresented terms in the biological process category were found to be significantly involved with processes related to phenylpropanoid biosynthesis (e.g., 'phenylpropanoid biosynthetic process' and 'phenylpropanoid metabolic process') and sucrose metabolism (e.g., 'sucrose metabolic process', 'glucose metabolic process', 'glucan metabolic process', and 'polysaccharide metabolic process' [Supplementary Table S4]). The DEGs in GO terms related to phenylpropanoid biosynthesis and sucrose metabolism are critical candidate genes that may play key roles in BABA-elicited defense responses against $B$. cinerea infection in grapes.

\section{Functional classifications of DEGs.}

All DEGs identified in the pairwise comparisons of $10 \mathrm{mM}$ BABA-vs-control, $100 \mathrm{mM}$ BABA-vs-control, and $10 \mathrm{mM}$ BABA-vs-100 mM BABA were annotated in the Eukaryotic Clusters of Orthologous Groups (KOG) database and were functionally classified into 25 categories. Among the 25 KOG categories, the orthology cluster for 'signal transduction mechanisms' (89 DEGs, $20.55 \%$ ) predominated in the $10 \mathrm{mM}$ BABA-vs-control pairwise comparison, followed by 'general function prediction only' (53 DEGs, $12.24 \%$ ) and 'posttranslational modification, protein turnover, chaperones' (47 DEGs, 10.85\%) (Fig. 5E). In addition, 'general function prediction only' was the most highly enriched category in $100 \mathrm{mM}$ BABA-vs-control pairwise comparison, with 113 DEGs, accounting for $16.37 \%$ of the annotations (Fig. 4E). A large proportion of DEGs were enriched in the 'signal transduction mechanisms' (22 DEGs, 13.50\%) and 'carbohydrate transport and metabolism' (16 DEGs, 9.82\%) categories in the $10 \mathrm{mM}$ BABAvs-100 mM BABA pairwise comparison (Fig. 4E).

Regarding Kyoto Encyclopedia of Genes and Genomes (KEGG) pathway enrichment, 108, 106, and 51 pathways were annotated from the DEGs in $10 \mathrm{mM}$ BABA-vs-control, $100 \mathrm{mM}$ BABA-vs-control, and $10 \mathrm{mM}$ BABA-vs-100 mM BABA pairwise comparisons, respectively (Supplementary Table S3). Notably, the most significant pathways were 'metabolic pathways' (37 DEGs) and 'starch and sucrose metabolism' (35 DEGs), followed by 'biosynthesis of secondary metabolites' (27 DEGs), 'phenylpropanoid biosynthesis' (26 DEGs) and 'fructose and mannose metabolism' (23 DEGs) in the $10 \mathrm{mM}$ BABA-vs-control pairwise comparison. In addition, 161 DEGs were assigned to several main functional terms, including 'metabolic pathways', 'phenylpropanoid biosynthesis', 'biosynthesis of secondary metabolites', 'flavonoid biosynthesis', and 'sucrose metabolism', in the $100 \mathrm{mM}$ BABA-vs-control pairwise comparison. The DEGs identified in the $10 \mathrm{mM}$ BABA-vs$100 \mathrm{mM}$ BABA pairwise comparison were assigned to 12 significant KEGG pathways, and most of the DEGs were enriched in 'phenylpropanoid biosynthesis', 'starch and sucrose metabolism', and 'metabolic pathways'. Remarkably, the dominant pathways were 'phenylpropanoid biosynthesis' (ko00940) and 'starch and sucrose metabolism' (ko00500) in the above three pairwise 
comparisons, implying significant differences in the physiological and biochemical responses involved in phenylpropanoid biosynthesis as well as sucrose metabolism in the process of BABA-IR against $B$. cinerea in grape berries.
Comparative transcriptomic profiling of DEGs involved in phenylpropanoid and sucrose metabolism pathways.

To visualize the dominant metabolic events related to phenylpropanoid biosynthesis and sucrose metabolism, the associated
A

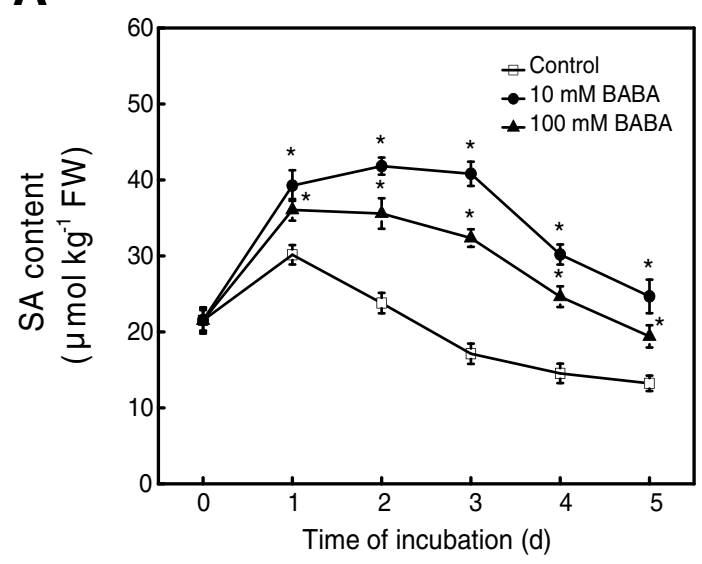

C

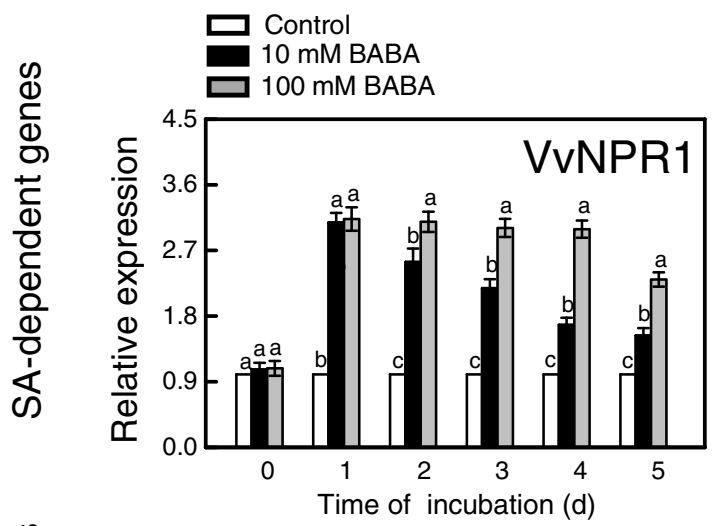

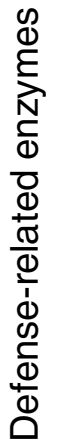

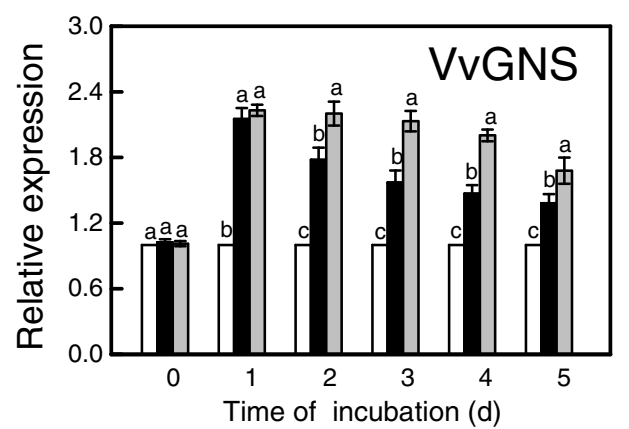

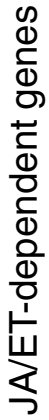

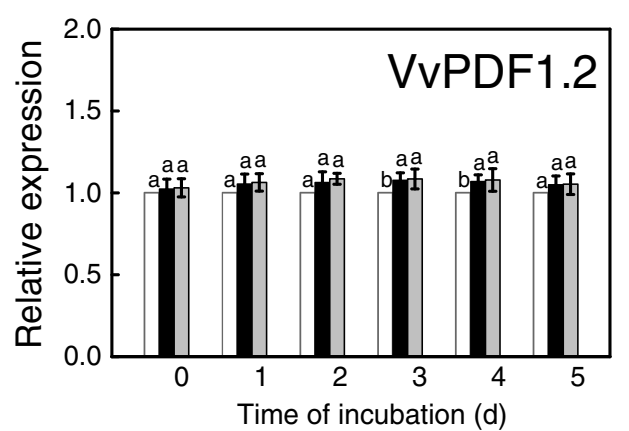

B
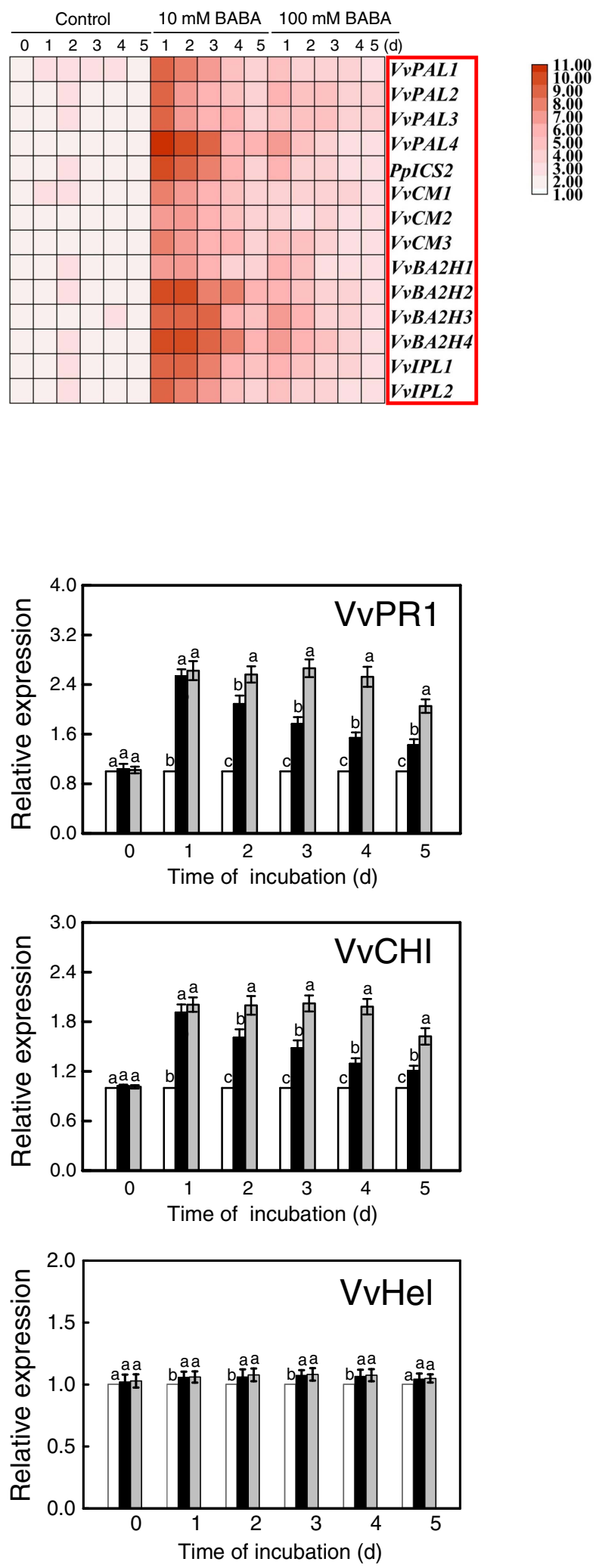

Fig. 3. A, $\beta$-Aminobutyric acid (BABA) treatments affected the salicylic acid (SA) content and $\mathbf{B}$, the expression levels of $\mathrm{SA}$ biosynthesis genes ( $V v P A L s, V v I C S 2$, $V v C M s, V v B A 2 H s$ and $V v I P L s)$ and $\mathbf{C}$, pathogenesis-related $(P R)$ genes ( $V v N P R 1, V v P R 1, V v G N S, V v C H I, V v P D F 1.2$, and $V v H e l)$ in grape berries. SA content and $P R$ genes expression are recorded as the mean \pm standard error $(\mathrm{SE})$ of triplicate assays. Statistical vertical bars represent $\mathrm{SE}$. Different letters indicate significant $(P<0.05)$ differences among treatments. Mitochondrial RNA (mRNA) transcripts of SA biosynthesis genes were profiled by quantitative PCR and were visualized via a heatmap. 
KEGG pathways were integrated and are depicted in Figure 5A. The $\log _{2}$ fold change $\left(\log _{2} \mathrm{FC}\right)$ values of the DEGs encoding the key enzyme genes from phenylpropanoid biosynthesis, such as phenylalanine ammonium lyases (PALs), 4-coumarate coenzymes (4CLs), cinnamate-4-hydroxylase (C4H), trans-cinnamate 4-monooxygenases (CYP73As), and stilbene synthases (STSs), identified in the above-mentioned pairwise comparisons discovered that these DEGs exhibited similar expression trends and all were significantly upregulated in 10 or $100 \mathrm{mM}$ BABA-treated grape berries relative to the controls (Fig. 5B). Treatment of grapes with $10 \mathrm{mM}$ BABA slightly downregulated the transcript profiles of these DEGs involved in phenylpropanoid biosynthesis in comparison with grapes subjected to $100 \mathrm{mM}$ BABA treatment. In addition, the transcript levels of several DEGs encoding enzymes involved in sucrose metabolism, comprising sucrose synthases (SSs), sucrose phosphate synthases (SPSs), sucrosephosphatases (SPPs), UDPases, acid invertases (AIs), and neutral invertases (NIs) were upregulated in $10 \mathrm{mM}$ BABA-treated grape berries compared with the controls and the $100 \mathrm{mM}$ BABA-treated berries. In addition to UDPases, other DEGs showed downregulated transcript levels in the pairwise comparison of 100 mM BABA-vs-control (Fig. 5B).

Validation of RNA-seq data by quantitative PCR (qPCR).

To validate the expression of the DEGs encoding critical enzymes in the phenylpropanoid and sucrose metabolism

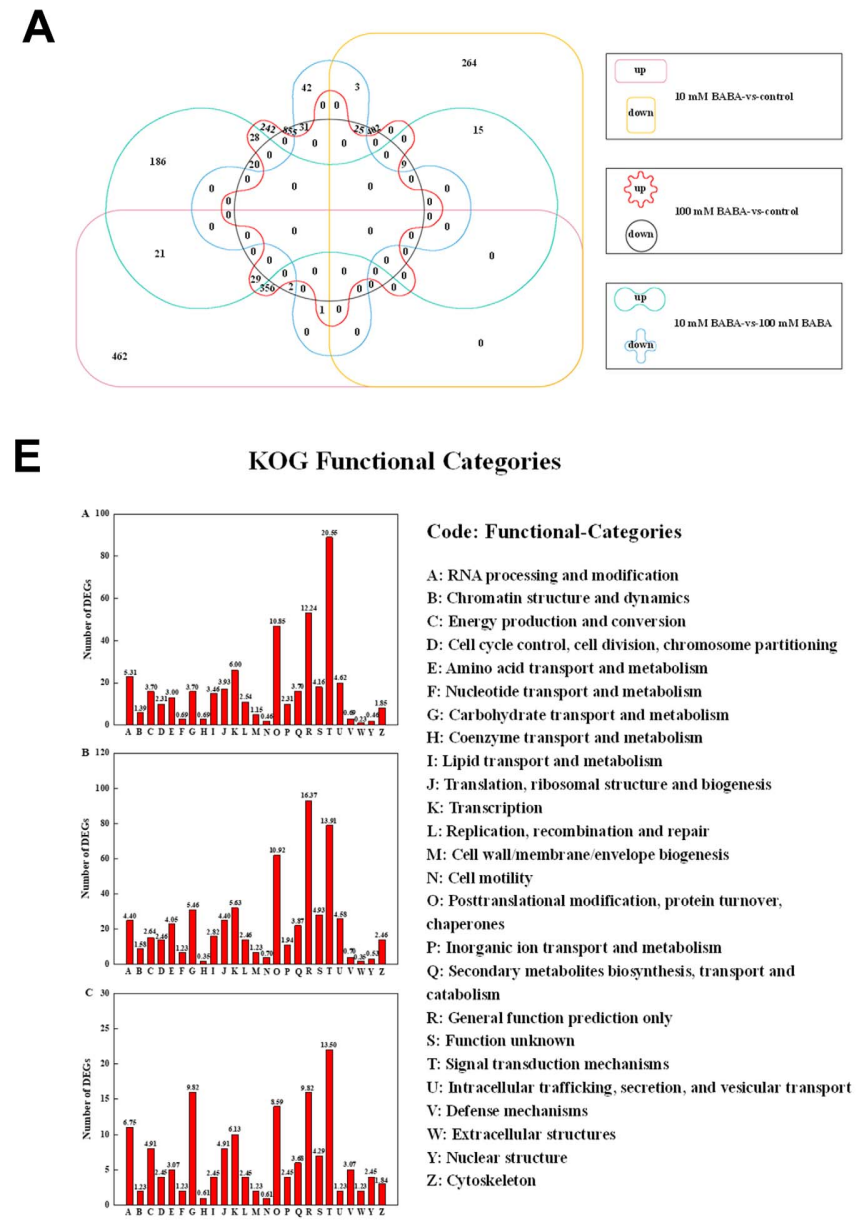

pathways identified in the Illumina sequencing analysis, we measured their expression levels by qPCR and further compared their $\log _{2} \mathrm{FC}$ values among the three pairwise comparisons (Supplementary Fig. S1A). The expression patterns of these DEGs were in accordance with the RNA-seq data and a determinable correlation $\left(R^{2}=0.857\right)$ was found between them (Supplementary Fig. $\mathrm{S} 1 \mathrm{~B})$.

\section{Nuclear-localized $V v M Y B 44$ interacts with $V v N P R 1$ in vivo.}

The combined phylogenetic tree of the AtMYB44 and $V$. vinifera MYB proteins showed that the VvMYB44 protein was clustered into the same branch as AtMYB44; one R2 HTH and one R3 HTH comprising approximately 50 amino acid residues, respectively, were shared between the two MYB proteins (Supplementary Fig. S2). AtMYB44 positively modulates SA-dependent defense in Arabidopsis (Shim et al. 2013; Zou et al. 2013), and the transcript of the gene ortholog of AtMYB44 from $V$. vinifera ( $V v M Y B 44)$ was significantly induced by $B$. cinerea inoculation and BABA treatments (Fig. 6A), suggesting the possible participation of R2R3-type $V v M Y B 44$ in BABA-elicited defense against fungal pathogens in grape berries. As shown in Figure 6B, the control construct harboring the pEAQ-GFP (green fluorescemt protein) cassette exhibited expression in the nucleus and cytoplasm of onion bulb epidermis cells; in contrast, the pEAQ- $V v M Y B 44-\mathrm{GFP}$ fusion protein was localized exclusively within the nucleus, as
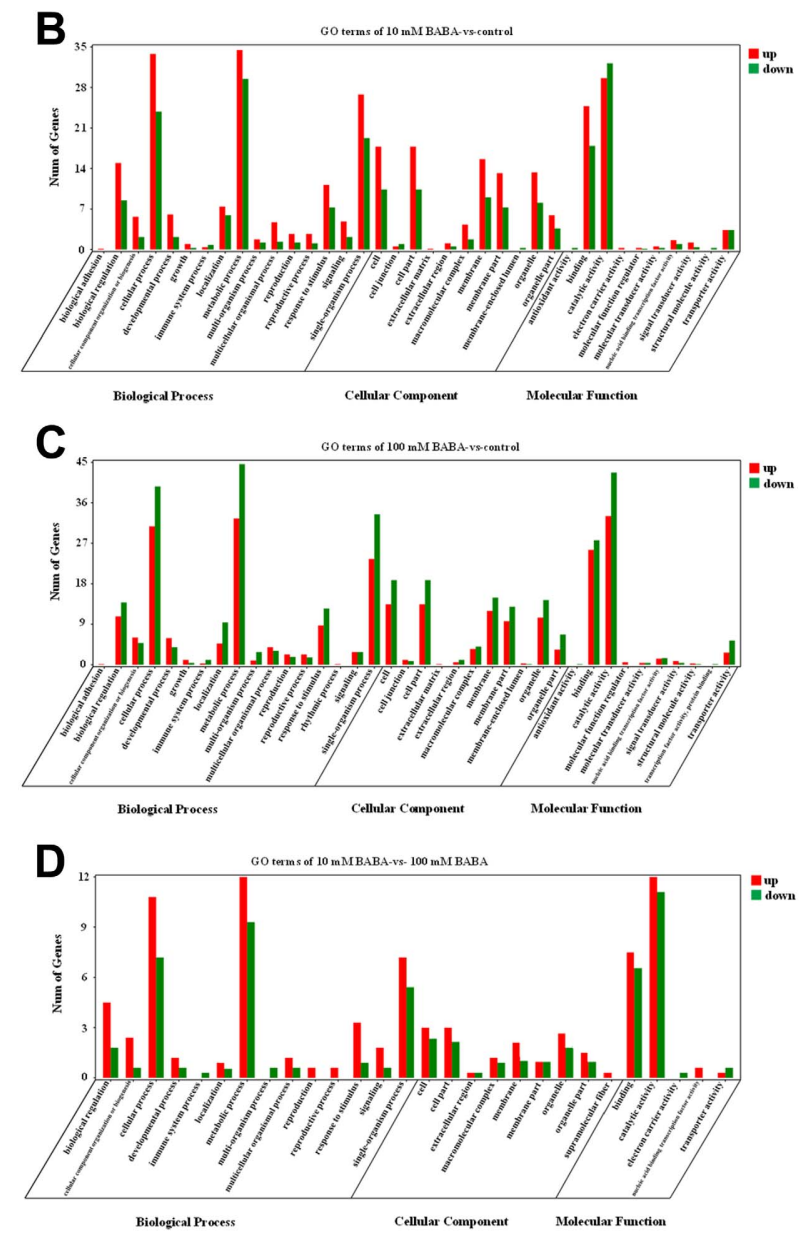

Fig. 4. Transcriptome profiling analysis of $\beta$-aminobutyric acid (BABA)-induced resistance against the fungal pathogen Botrytis cinerea in postharvest grape berries. A, A Venn diagram of differentially expressed genes (DEGs) across three pairwise comparisons (10 mM BABA-vs-control, $100 \mathrm{mM}$ BABA-vs-control, and $10 \mathrm{mM}$ BABA-vs-100 mM BABA). B through D, Histograms of gene ontology functional classifications of DEGs among the pairwise comparisons of $10 \mathrm{mM}$ BABA-vs-control, $100 \mathrm{mM}$ BABA-vs-control, and $10 \mathrm{mM}$ BABA-vs-100 mM BABA, in which the red and green frames represent up- and downregulated DEGs, respectively. E, Eukaryotic Clusters of Orthologous Groups (KOG) classifications in grapevine. Approximately 433 of the 822 DEGs, 568 of the 933 DEGs, and 163 of the 229 DEGs with nonredundant hits from 10 mM BABA-vs-control, 100 mM BABA-vs-control, and 10 mM BABA-vs-100 mM BABA pairwise comparisons, respectively, were grouped into $25 \mathrm{KOG}$ classifications; the percentage of DEGs is presented at the top of each column. 
highlighted by 4',6-diamidino-2-phenylindole (DAPI) staining. In the yeast two-hybrid $(\mathrm{Y} 2 \mathrm{H})$ assay, the interaction between $V v M Y B 44$ and $V v N P R 1$ activated the reporter genes HIS3, ADE2, and MEL1, since AH109 cells transfected with BD-VvMYB44 and $\mathrm{AD}-V v N P R 1$ were observed to form colony clusters on synthetic dropout plates (TDO [SD/-Trp/-Leu/-His] and QDO [SD/-Trp/ -Leu/-His/-Ade]) within 4 to 6 days, and these colonies displayed a white to blue color change on X- $\alpha$-gal plates (Fig. 6C). Furthermore, this interaction between $V v M Y B 44$ and $V v N P R I$ was confirmed in planta through a coimmunoprecipitation (Co-IP) assay (Fig. 6D).

\section{$V v M Y B 44$ can directly activate the transcripts of enzyme-encoding genes involved in phenylpropanoid and sucrose metabolism.}

A previous study showed that most MYB proteins enhance the transcripts of their downstream genes by binding to MBSs such as MBS I [CNGTT(A/G)] and MBS II [C(G/T)T(A/ T)GTT(A/G)] in their promoters (Romero et al. 1998). We randomly characterized the promoter regions of the enzymeencoding genes involved in phenylpropanoid and sucrose metabolism, comprising SS2/SPS3/SPP1/UDPase3/AI/NI/PAL/4CL/ $C 4 H / C Y P 73 A / S T S 1$, and at least one putative MBS was identified among them (Supplementary Data S1). Notably, VvMYB44 bound directly to the MBS I or MBS II site within the $S S 2$, SPS3, SPP1, UDPase3, AI, NI, PAL, 4CL, C4H, CYP73A, or STS1 promoters in the yeast one-hybrid $(\mathrm{Y} 1 \mathrm{H})$ assay (Fig. 7A). When the SS2, SPS3, SPP1, UDPase3, AI, NI, PAL, 4CL, C4H, $C Y P 73 A$, or STS1 Pro-LUC (luciferase) reporter construct was coinfiltrated with $V v M Y B 44$, the LUC/REN (Renilla luciferase) ratio was more intensely increased and was markedly higher than that of the empty control $(35: \because 62-S K+$ Pro::LUC) (Fig. $7 \mathrm{~B})$. The $\mathrm{Y} 1 \mathrm{H}$ and dual-LUC reporter (DLR) results indicated that $V v M Y B 44$ can directly transactivate the expression of enzyme-encoding genes from phenylpropanoid and sucrose metabolism via MBSs.

\section{Heterologous expression of $V v M Y B 44$ potentiates the resistance of transgenic Arabidopsis to fungal pathogen.}

As previously recorded, AtMYB44 exerts a vital effect on plant disease resistance (Shim et al. 2013; Zou et al. 2013). VvMYB44 served as the orthologous gene of AtMYB44 isolated from Vitis vinifera, and both of them were identified as R2R3-type MYBs (Supplementary Fig. S2). To evaluate the effectiveness of $V v M Y B 44$ in the plant defense response to fungal pathogens, transcripts encoding the PR NPR1 and PR1 proteins in B. cinerea-infected transgenic Arabidopsis overexpressing VvMYB44 and wild-type (WT) (Columbia-0 [Col-0]) plants were recorded. As

\section{A}

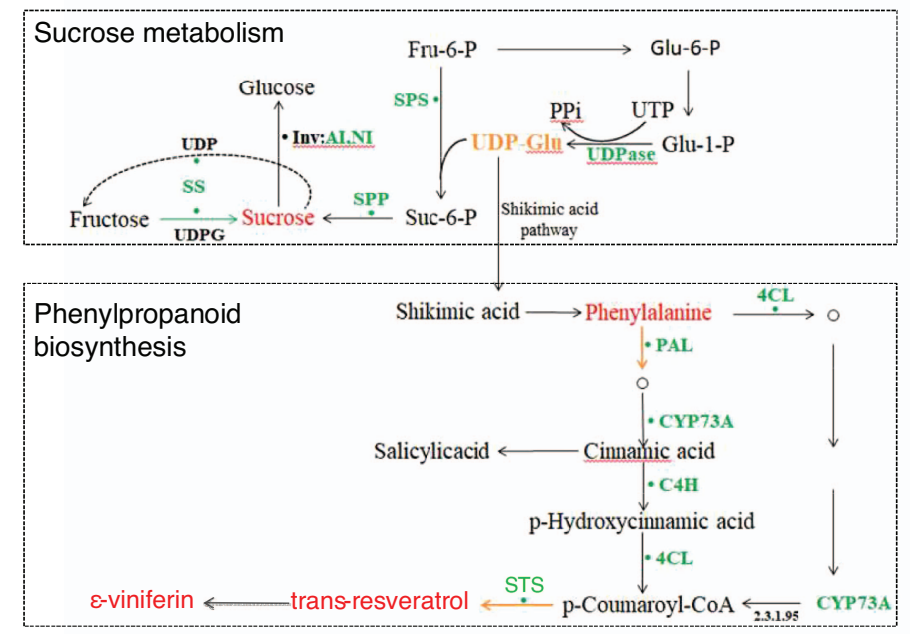

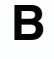

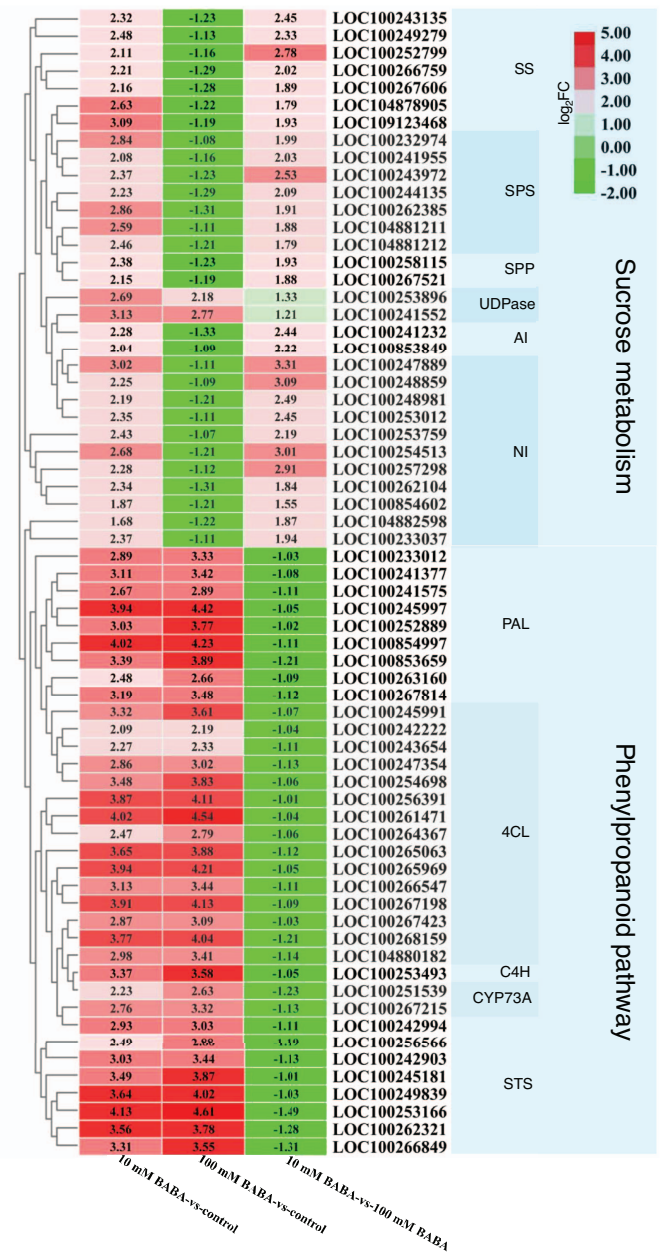

Fig. 5. A, Scheme of sucrose metabolism and the phenylpropanoid biosynthetic pathway and the involved regulatory differentially expressed genes (DEGs) identified in the grapevine transcriptome. SS = sucrose synthase, SPP = sucrose-phosphatase, AI = acid invertase, NI = neutral invertase, SPS = sucrose phosphate synthase, UDPase $=$ uridine diphosphatase, $\mathrm{UDPG}=\mathrm{UDP}$-glucose, $4 \mathrm{CL}=4$-coumarate coenzyme $\mathrm{A}$ ligase, $\mathrm{PAL}=$ phenylalanine ammonium lyase, $\mathrm{CYP73 \textrm {A }}=$ trans-cinnamate 4-monooxygenase, $\mathrm{C} 4 \mathrm{H}=$ cinnamate-4-hydroxylase, $\mathrm{CHS}=$ chalcone synthase, $\mathrm{STS}=$ stilbene synthase, Glu-6-p = glucose-6-phosphate, Glu-1-p = glucose-1-phosphate, UTP = uridine triphosphate, PPi = pyrophosphate, Fru-6-p = fructose 6-phosphate, Suc6-p = sucrose-phosphate. B, Heat map of DEGs involved in phenylpropanoid and sucrose metabolism among the pairwise comparisons of $10 \mathrm{mM}$ $\beta$-aminobutyric acid (BABA)-vs-control, $100 \mathrm{mM}$ BABA-vs-control, and $10 \mathrm{mM}$ BABA-vs-100 mM BABA. 
A

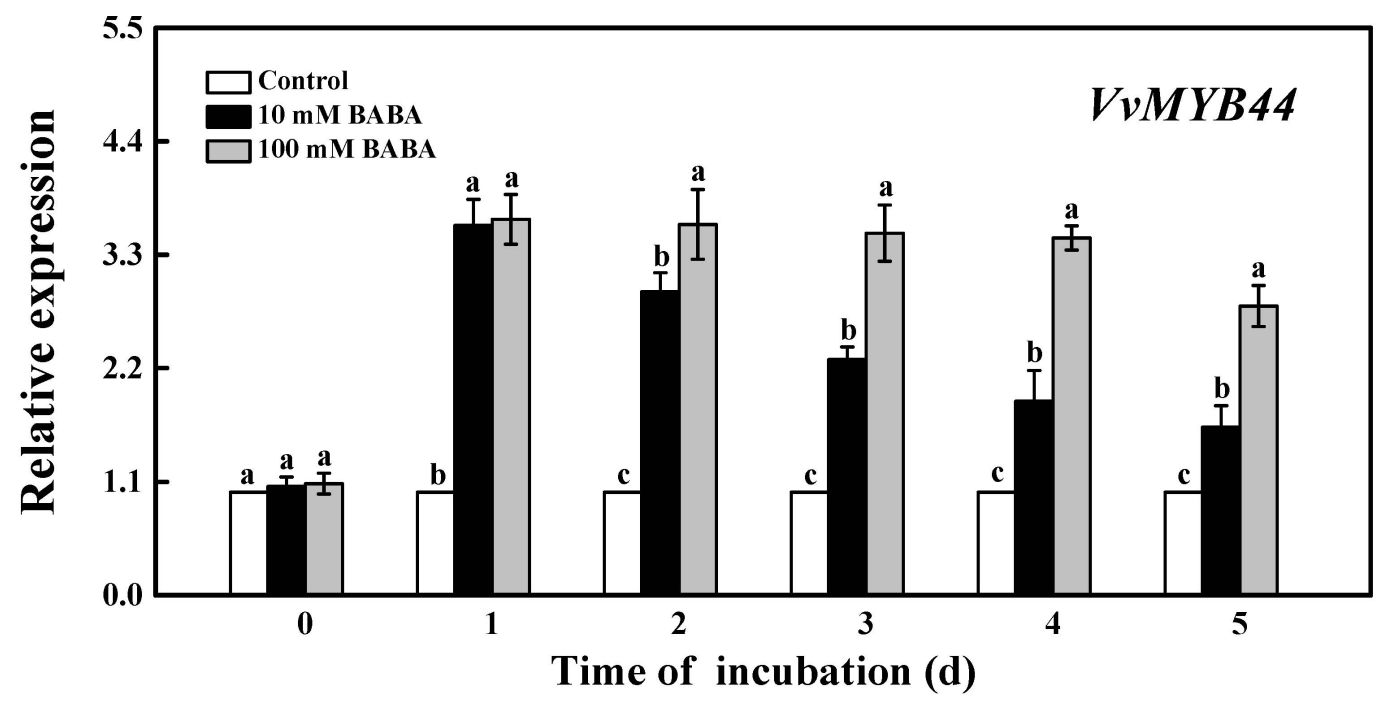

B

Bright Field
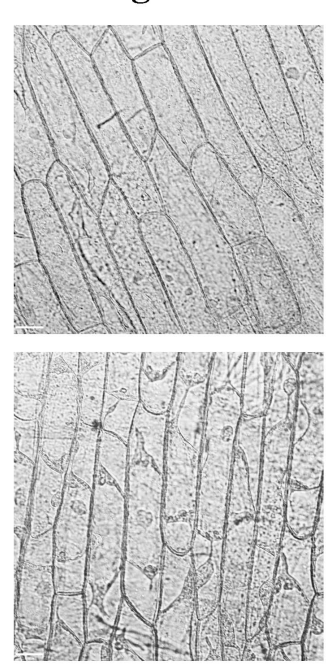

GFP
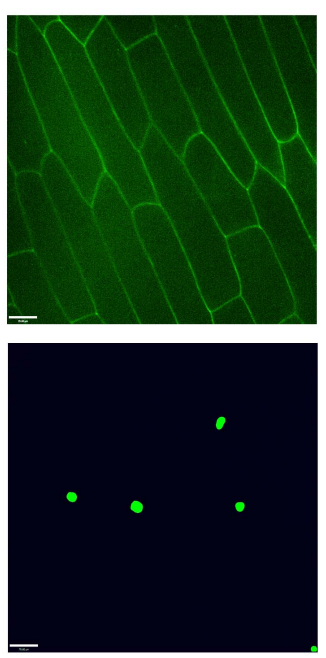

DAPI
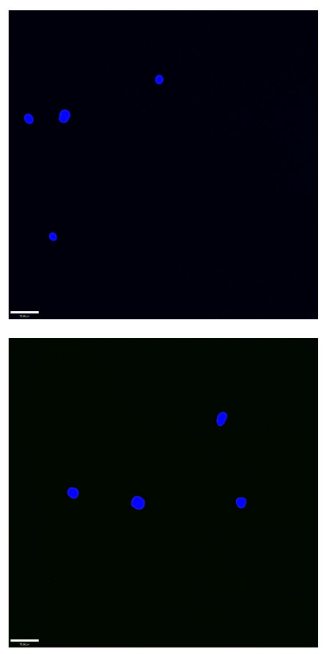

Merged
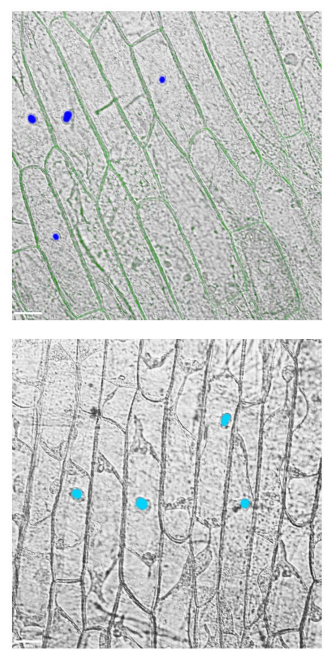

pEAQ-VvMYB44-GFP
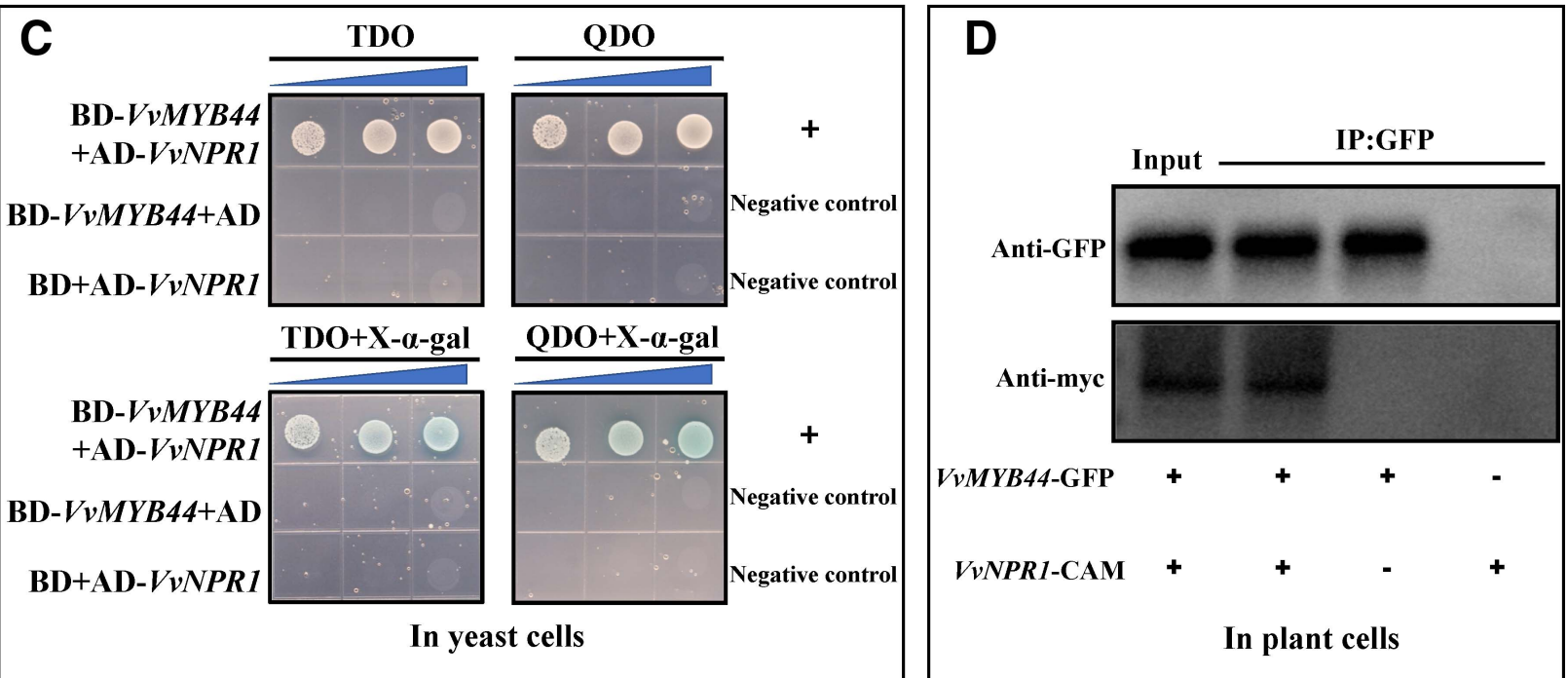

Fig. 6. Nuclear-localized $V v M Y B 44$ physically interacted with NPR1 in vivo. A, Changes in transcript levels of $V v M Y B 44$ in grapes after inoculation with Botrytis cinerea at 1-day intervals. B, Nuclear localization of the pEAQ-VvMYB44-GFP (green fluorescent protein) recombinant protein, in which the nuclei were highlighted by 4',6-diamidino-2-phenylindole (DAPI) staining (bars $=70 \mu \mathrm{m})$. C, The yeast two-hybrid assay (Y2H) uncovered the direct interaction between $V v M Y B 44$ and $V v N P R 1$. The right-angle triangles represent the absorbance of yeast at $600 \mathrm{~nm}$ in a 10 -fold dilution series, from $10^{-2}$ to 1 absorbance. D, Coimmunoprecipitation (Co-IP) results supported the VvMYB44 and VvNPR1 interaction in tobacco (Nicotiana tabacum). Both the $\mathrm{Y} 2 \mathrm{H}$ and Co-IP experiments were conducted twice with similar results and all the presented data derive from the second repeat experiment. 
seen in Figure 8C, the mitochondrial RNA (mRNA) accumulations of NPRI and PRI were significantly elevated in the transgenic Arabidopsis lines (OE-4, OE-7, and OE-13) overexpressing the coding gene of grape $M Y B 44$ at both 3 and 6 days postinfection (dpi), as compared with the WT plants. Additionally, overexpression of $V v M Y B 44$ also extremely enhanced the transcripts of a set of phenylpropanoid and sucrose metabolism-related genes, especially SS2, SPS3, SPP1, UDPase3, PAL, 4CL, C4H, $C Y P 73 A$, and STS1. Moreover, higher transcripts of these genes were observed after treating the transgenic lines with the BABA formulation. To visualize the state of pathogen growth and cell death in WT and VvMYB44-transgenic plants, we further imaged $B$. cinerea-infected leaves after staining with trypan blue. Almost all of the cells in WT leaves degenerated and died, as visibly obtained by staining with trypan blue; however, the lesion areas and necrotic features were totally lacking in OE-4, OE-7, and OE-13 (Fig. 8D). Combined with the above data, these results confirmed that overexpression of $V v M Y B 44$ is capable of alleviating cell damage and disease progression in transgenic Arabidopsis but did not impair the growth of the transgenic plants (Fig. 8B).

\section{DISCUSSION}

Plants are sessile organisms that are inevitably exposed to a combination of biotic and abiotic stresses (Khare et al. 2020). The priming molecule BABA applied in the present study is a verified elicitor of plant defense by mediating the combined actions of diverse phytohormonal signaling networks and thus potentiating the plant capacity to defend against certain stresses (Cohen et al. 2016; Thevenet et al. 2017). Our current work demonstrated that the defense capacity against the fungal pathogen $B$. cinerea elicited by $10 \mathrm{mM}$ BABA contributes to an approximately $26 \%$ reduction in the postharvest decay of grapes, but the decay incidence (DI) of $100 \mathrm{mM}$ BABA-treated grapes was only slightly lower than that of grapes treated with $10 \mathrm{mM}$ BABA (Fig. 1A and B). In parallel, BABA treatments lowered the respiration rate and ET production, indicating that the BABA efficiently delayed physiological senescence, as confirmed by lower MDA content in the BABA-treated samples compared with the controls (Fig. 1D through F). Normally, a strong burst of $\mathrm{H}_{2} \mathrm{O}_{2}$ functions as a physiological marker in the early defense responses of many plants (Mazid et al. 2011). $\mathrm{H}_{2} \mathrm{O}_{2}$ is a proposed elicitor of the hypersensitive response and is associated with the reinforcement of the cell wall and transcripts of defense-related genes and activation of resistance during the expression of plant disease resistance (Levine et al. 1994; O'Brien et al. 2012). Furthermore, Dubreuil-Maurizi et al. (2010) have proved that $\mathrm{H}_{2} \mathrm{O}_{2}$ accumulation is tightly related to BABA-elicited defense responses in plants. $\mathrm{An} \mathrm{H}_{2} \mathrm{O}_{2}$ burst in BABA-treated grapes was also accompanied by the upregulation of a set of $P R$ genes (Figs. $1 \mathrm{C}$ and $3 \mathrm{C}$ ), showing the crucial role of $\mathrm{H}_{2} \mathrm{O}_{2}$ in BABA-mediated defense in grapes. Upon $B$. cinerea inoculation, BABA treatments apparently reinforced the transcripts of SA-dependent genes, such as NPRI and PRI, and two defense-related enzymes, namely, $V v G N S$ and $V v C H I$. Specifically, the peak expression profiles of $P R$ protein or genes, including $V v N P R 1, V v G N S, V v C H I$, and SA-inducible $V v P R 1$, in the berries treated with BABA at $10 \mathrm{mM}$ were observed

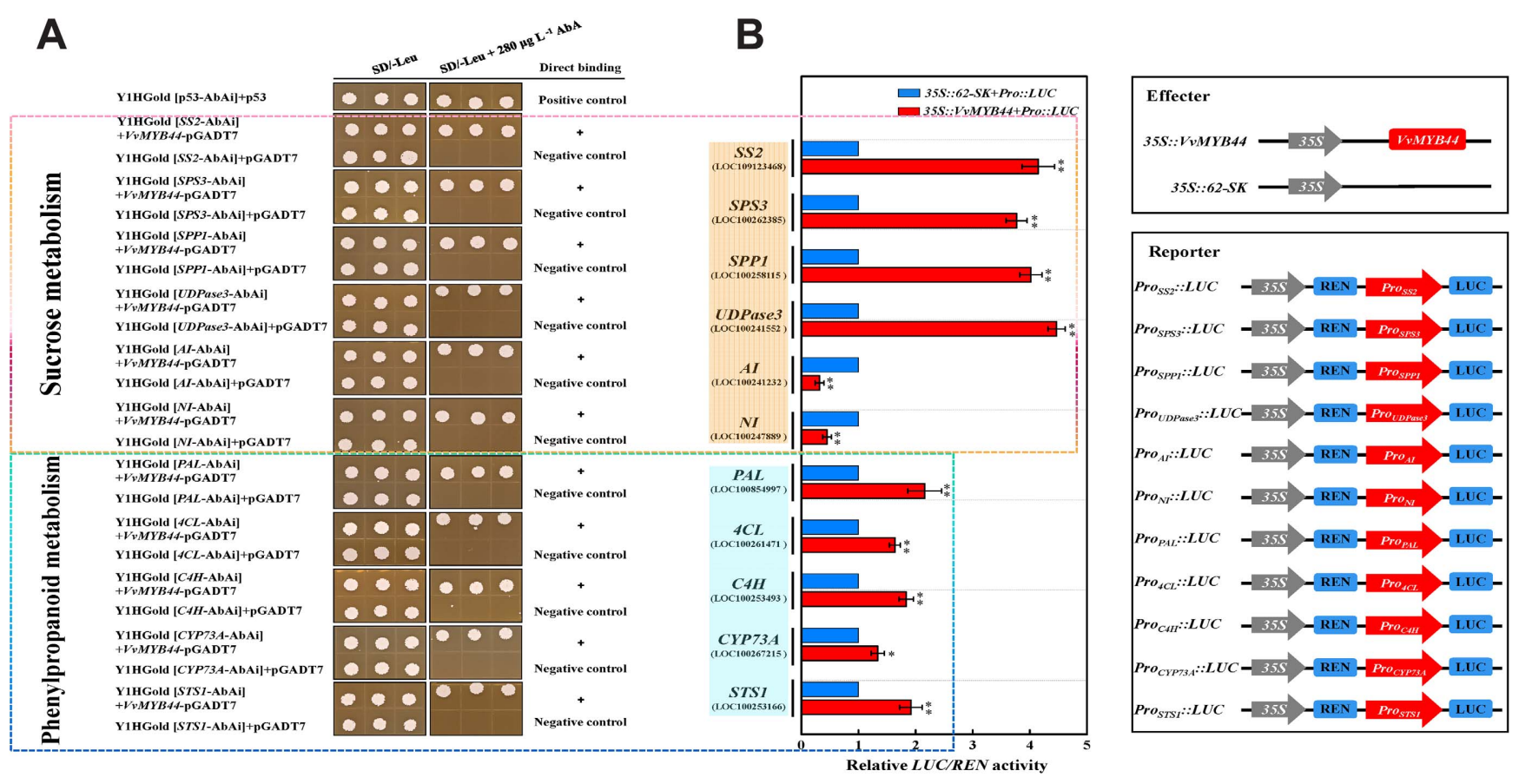

Fig. 7. VvMYB44 can bind to MYB-binding sites (MBSs) within the promoter sequences of enzyme-encoding genes related to phenylpropanoid and sucrose metabolism and activate their transcripts. A, The direct binding of VvMYB44 to MBSI or MBSII within the promoter regions of sucrose synthase SS2, sucrose phosphate synthase SPS3, sucrose-phosphatase SPP1, UDPase3, AI (acid invertase), NI (neutral invertase), PAL (phenylalanine ammonium lyase), 4CL (4-coumarate coenzyme), C4H (cinnamate-4-hydroxylase), CYP73A (trans-cinnamate 4-monooxygenase), and stilbene synthase STS1 was determined based on the ability of Y1HGold ( VvSS2/VvSPS3/VvSPP1/VvUDPase $3 / V v A I / V v N I / V v P A L / V v 4 C L / V v C 4 H / V v C Y P 73 A /$ $V v S T S 1-A b A i)+V v M Y B 44-$ pGADT7 to grow on synthetic dropout (SD)/-Leu in the presence of $280 \mu \mathrm{g}$ of AbA per liter. B, A dual-luciferase (LUC) reporter (DLR) assay for the VvMYB44-mediated transactivation of the promoters of SS2, SPS3, SPP1, UDPase3, AI, NI, PAL, 4CL, C4H, CYP73A, and STS1. Transactivations are mirrored by ratios of LUC to Renilla luciferase, and the value for the empty plasmid combined with the promoters of SS2, SPS3, SPP1, UDPase3, AI, NI, PAL, 4CL, C4H, CYP73A, or STS1 was set to 1 for calibration of the ratio. Data represent the mean \pm standard error of nine independent repeats from each experiment. Asterisks $\left(^{* *}\right)$ indicate significant differences among samples $(P=0.01)$ Both the yeast one-hybrid and DLR experiments were conducted twice with similar results and all the presented data derive from the second repeat experiment. 

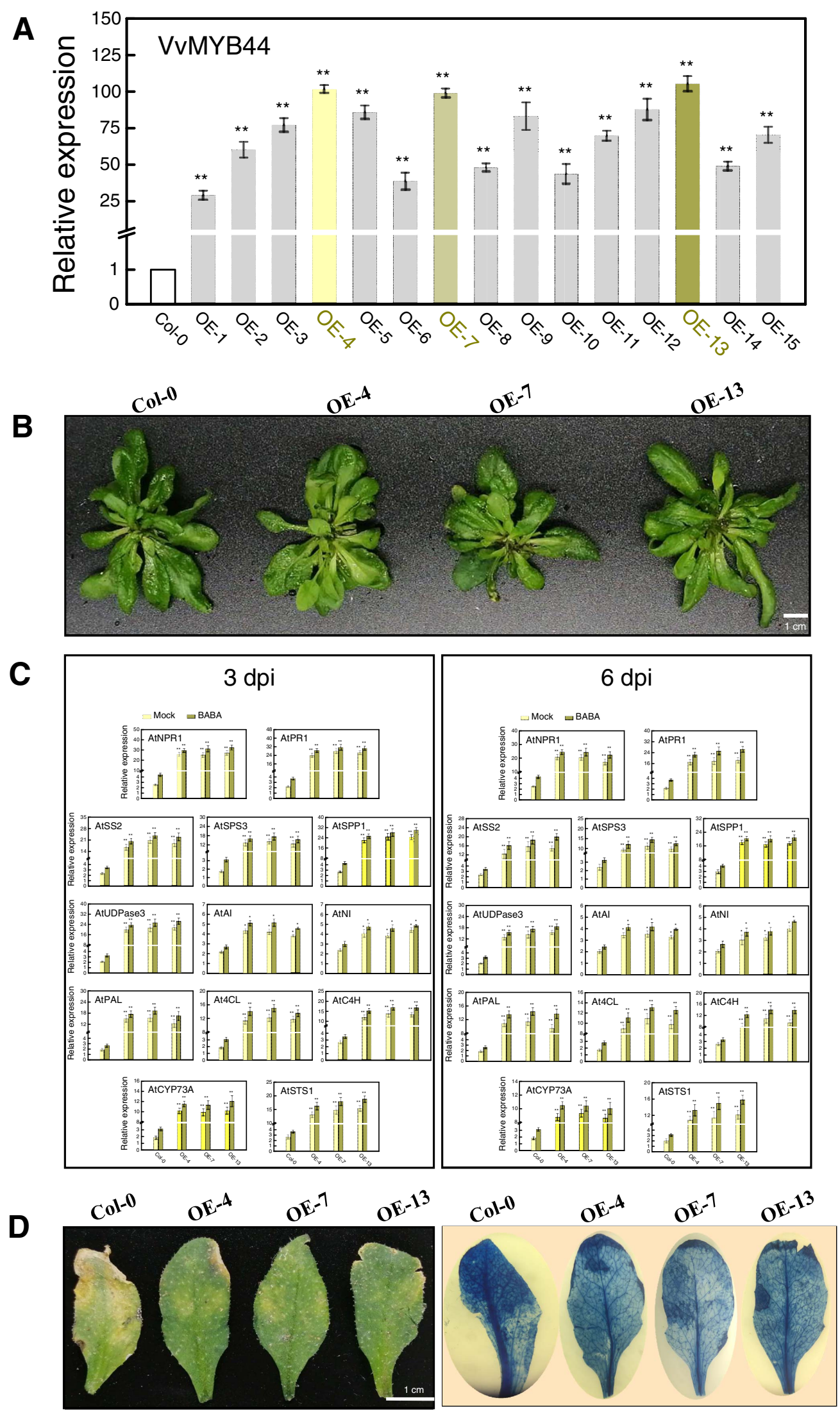

Fig. 8. Overexpression of $V v M Y B 44$ activates the expression of pathogenesis-related $(P R)$ and sucrose and phenylpropanoid metabolism-related genes in Arabidopsis leaves and suppresses apoptotic cell death and enhances fungal resistance in transgenic Arabidopsis. A, VvMYB44 transcript in wild-type and transgenic plants. Transcript abundance was calculated relative to AtActin 2 and recorded as the mean \pm standard error. Asterisks $\left({ }^{*}\right)$ represent significant $(P<0.01)$ differences between wild-type (WT) and transgenic plants. B, Overexpression of VvMYB44 exerted little negative effect on the growth phenotype of transgenic Arabidopsis. Bar $=1 \mathrm{~cm}$. C, Transcripts of $P R$ genes and phenylpropanoid and sucrose metabolism-related genes in WT and transgenic Arabidopsis plants (OE-4, OE-7, and OE-13) challenged with Botrytis cinerea at 3 and 6 days postinfection (dpi) with or without $\beta$-aminobutyric acid treatment. D, Disease symptoms and necrotic areas in Col-0 WT and VvMYB44-overexpressing leaves were determined by trypan blue staining after B. cinerea inoculation (6 dpi). 
approximately 1 day after inoculation with the fungal spore suspensions, followed by a continuous substantial decrease (Fig. 3C). In contrast, with the exception of a slight decrease on the fifth day, the transcript levels of $P R$ genes in grapes treated with $100 \mathrm{mM}$ BABA fluctuated around the peak levels (Fig. 3C). As shown in our previous studies addressing the dose-dependent mode of BABA-IR (Li et al. 2021a; Wang et al. 2016), the grape defensive trait activated by $100 \mathrm{mM}$ BABA was mechanistically attributed to direct defense and priming defense elicited by the lower BABA concentration of $10 \mathrm{mM}$. The defensive capacity, mainly reflected by the DI index, the transcript levels of $P R$ genes and the accumulation of stilbene phytoalexins in $100 \mathrm{mM}$ BABA-induced direct defense did not differ greatly from that in the priming defense elicited by BABA treatment at $10 \mathrm{mM}$, while $100 \mathrm{mM}$ BABA-induced direct defense exerted a detrimental effect on soluble sugar accumulation in grapes (Fig. 2). Hence, the hyperactivation of resistance in the direct defense mode elicited by $100 \mathrm{mM}$ BABA not only could not completely abolish the development of gray mold disease symptoms in grape berries but also imposed a certain metabolic cost. Remarkably, $10 \mathrm{mM}$ BABAelicited priming resistance showed an equivalent protective effectiveness to direct resistance and did not arouse an excessive consumption in soluble sugars. Thus, the priming defense induced by BABA treatment at $10 \mathrm{mM}$ represents an efficient strategy for activating host resistance to fungal pathogen $\mathrm{B}$. cinerea infection in grapes.

Rapid and extensive transcriptional reprogramming upon fungal pathogen recognition is the key step in the deployment of plant defenses during phytopathogen interactions (Lyons et al. 2015; Kim et al. 2020; Panda et al. 2019; Smith et al. 2014). We monitored the transcriptional reprogramming activated by BABA treatment and $B$. cinerea inoculation during the early establishment of the plant-fungal interaction and probed the potential role of an R2R3-type MYB TF gene, $V v M Y B 44$, in this process. We observed that the transcript levels of hundreds of host genes were differentially altered within one day, indicating that specific signaling between the pathogen and individual host cells appeared one day after exposure to the fungal spores on the pericarp surface, and this process was clearly affected by BABA treatments at different concentrations. Through Illumina HiSeq 2500 paired-end sequencing, a total of 1,589 genes were differentially expressed in $10 \mathrm{mM}$ BABA-primed berries, including 871 upregulated $(54.81 \%)$ and 718 downregulated genes (45.19\%), while $100 \mathrm{mM}$ BABA induced $656(32.80 \%)$ up- and 1,344 (67.20\%) downregulated DEGs when compared with the control grapes, indicating that transcriptional activators might play an essential role in the defensive activities of the 10 $\mathrm{mM}$ BABA-treated grapes against the fungal pathogen $\mathrm{B}$. cinerea. Moreover, most of the identified DEGs in the $10 \mathrm{mM}$ BABA-vs-control, $100 \mathrm{mM}$ BABA-vs-control, and $10 \mathrm{mM}$ BABA-vs-100 mM BABA pairwise comparisons were significantly annotated to GO terms related to phenylpropanoid biosynthesis (e.g., 'phenylpropanoid biosynthetic process' and 'phenylpropanoid metabolic process') and sucrose metabolism (e.g., 'sucrose metabolic process', 'glucose metabolic process', 'glucan metabolic process', and 'polysaccharide metabolic process') in the biological process category. These results provide key positional candidate genes for the discovery of novel regulators controlling BABA-elicited defense responses in grapes. MapMan analysis further revealed that the DEGs from the aforementioned pairwise comparisons were enriched in an average of 88 biological pathways, and most of them were dominantly linked to 'phenylpropanoid biosynthesis' (ko00940) and 'starch and sucrose metabolism' (ko00500) (Supplementary Table S3). In source tissues, the synthesis of UDPG can be derived from the displacement of a single pyrophosphate from uridine triphosphate by glucose-1-phosphate under the catalytic action of uridine diphosphatase (UGPase), which works in conjunction with SPP and SPS in the synthesis of sucrose (Li et al. 2014; Morkunas et al. 2011). Alternatively, SS is able to catalyze the conversion of UDPG and fructose into sucrose, and enzymes AI and NI can, in turn, mediate the hydrolysis of sucrose to produce glucose (Miron and Schaffer 1991). In addition, UGPase can integrate into the phenylpropanoid metabolism pathway via a UDPG -dependent group translocator by means of the shikimic acid pathway, yielding a series of antifungal triterpenes and stilbenes (Thom et al. 1986). Specifically, as Kleczkowski (1994) observed several decades ago, UDPG is a major glucosyl donor located at the branch point of the phenylpropanoid pathway and sucrose metabolism. As depicted by the heatmap in Figure 5, a large subset of enzyme-encoding genes related to sucrose metabolism, including SS, SPS, SPP, AI, and NI genes, were clearly upregulated to a certain extent from berries treated with $10 \mathrm{mM}$ BABA but were downregulated upon BABA treatment at $100 \mathrm{mM}$, compared with the controls. On the contrary, the grapes in the two BABA treatment groups showed similar increases in UDPase transcript levels, which were slightly higher in $10 \mathrm{mM}$ BABA-treated berries than in $100 \mathrm{mM}$ BABAtreated berries. Accordingly, BABA-induced UDPG accumulation was significantly more evident under $10 \mathrm{mM}$ BABA treatment than under $100 \mathrm{mM}$ BABA treatment (Fig. 2D). In terms of phenylpropanoid metabolism, the transcript levels of all critical enzyme-encoding genes were significantly increased by both BABA treatments, and $100 \mathrm{mM}$ BABA treatment resulted in higher transcript profiles than the $10 \mathrm{mM}$ BABA treatment. Therefore, the higher transcripts of enzyme-encoding genes involved in phenylpropanoid metabolism but the lower transcripts of enzymes related to sucrose metabolism in $100 \mathrm{mM}$ BABA-treated grapes indicated that more UDPG was transported into the phenylpropanoid metabolism pathway to increase the biosynthesis of antifungal trans-resveratrol and $\varepsilon$-viniferin, which impaired the production of soluble sugars in $100 \mathrm{mM}$ BABA-treated grapes. Notably, the transcript levels of all the enzyme-encoding genes related to phenylpropanoid and sucrose metabolism in $10 \mathrm{mM}$ BABA-primed berries were intermediate, which is conducive to maintaining the balance of sensory characteristics and the accumulation of antifungal stilbenes. Taken together, these results indicated that the chemical priming induced by a lower BABA dose did not cause any apparent quality or sensory loss on the premise that the accumulated levels of host-synthesized antifungal compounds were similar to those found in $100 \mathrm{mM}$ BABA-treated grapes upon challenge with the fungal pathogen $B$. cinerea; these results were in good accordance with the previous findings related to the defense of sugarcane against Leifsoniaxyli subsp. xyli (Fu et al. 2019).

The induction of plant host resistance to potential fungal pathogens involves the extensive transcriptional reprogramming of defense regulators. The R2R3-type MYB44 TFs participate in the expression of the defense response (Hieno et al. 2016; Qiu et al. 2019; Zou et al. 2013). Most molecular surveillance and genetic analyses of MYBs have been confined to the Arabidopsis model, but a few MYB proteins from grape have been identified. To isolate a similar factor related to grape defenses, we conducted a phylogenetic analysis of the MYB TF genes in $V$. vinifera and identified $V v M Y B 44$, which encodes a 2R-type MYB domain showing high sequence similarity to AtMYB44 (Supplementary Fig. S2). The expression of $V v M Y B 44$ was clearly induced after $B$. cinerea stimulation and BABA elicitation (Fig. 6A). In addition, we discovered that this nuclear-localized $V v M Y B 44$ factor can directly interact with the SA signaling regulatory gene NPRI in vivo, and transiently induced transcript of NPR1 in $10 \mathrm{mM}$ BABA-primed fruits was observed; meanwhile the SA-responsive 
PRl gene was also enhanced in these samples (Figs. 3 and 6). NPR1 presents high sensitivity and proximity to SA and thereby serves as a crucial regulator of SAR through the formation of NPR1 condensates (Zavaliev et al. 2020). Moreover, BABA can prime grapes to accumulate higher SA contents due to the BABAinduced transcripts of a set of SA biosynthesis-related genes, including $V v P A L$ genes, $V v I C S 2$, and $V v C M, V v B A 2 H$, and $V v I P L$ genes (Fig. 2A and B). In contrast, unlike the enhancement effect of $10 \mathrm{mM}$ BABA treatment on NPRI and $P R 1$ transcripts, the visible accumulation of JA or ET-dependent genes such as $V v P D F 1.2$ and $\mathrm{VvHel}$ did not occur (Fig. 3C). Thus, we deduced that BABAIR against $B$. cinerea in grape berries paralleled the primed onset of SA-dependent SAR reaction and that $V v M Y B 44$ participated in this process in an NPR1-dependent manner. These inferences were not only partially in accordance with previous findings indicating that $\mathrm{BABA}$ potentiates grape resistance against $B$. cinerea infection via a primed SAR response but were also sustained by the observation of decreased AtMYB44 expression in nprl, JA-insensitive coil, ET-insensitive etrl, or ABA-insensitive abi3-1 mutants (Jung et al. 2008, 2010; Wang et al. 2021; Zou et al. 2013). Several MYB TFs typically regulate downstream genes by binding to MYB recognition sequences within the promoter sequences of their target genes (Agarwal et al. 2006). The $\mathrm{Y} 1 \mathrm{H}$ and DLR results of the present study revealed that $V v M Y B 44$ is capable of binding the MBSs within the promoter regions of genes encoding enzymes associated with the sucrose metabolism pathway, including SS2, SPS3, SPP1, UDPase3, $\mathrm{AI}$, and $\mathrm{NI}$ and the phenylpropanoid metabolism pathway, such as PAL, 4CL, C4H, CYP73A, and STS1 and activating their expression (Fig. 7). Similar regulation of plant secondary metabolism by 2R-type MYBs, particularly the phenylpropanoid pathway, has also been pointed out previously (Bomal et al. 2014; Ma et al. 2018; Sablowski et al. 1994; Zhai et al. 2016). For example, three R2R3-MYB TFs from Arabidopsis (AtMYB32) and maize (ZmMYB31 and ZmMYB42) can directly bind to the caffeic acid $O$-methyltransferase promoter region (Fornalé et al. 2006; Preston et al. 2004); moreover, Antirrhinum AmMYB308 and AmMYB330 also retain the capacity to bind to the promoter sequences of $\mathrm{C} 4 \mathrm{H}, 4 \mathrm{CL}$, and cinnamyl alcohol dehydrogenase but, instead, repress their expression (Tamagnone et al. 1998). In addition, overexpressing $V v M Y B 44$ in transgenic Arabidopsis resulted in constitutive expression of the SA-responsive transcription coactivator NPR1 and SA-inducible PRI gene and increased the mRNA accumulation of sucrose and phenylpropanoid metabolismrelated genes, contributing to the lowered sensitivity of transgenic Arabidopsis to B. cinerea infection (Fig. 8). Therefore, we suggest that $V v M Y B 44$-mediated transcriptional regulation of another context-specific coregulator, NPR1, and a suite of downstream genes involved in sucrose metabolism and phenylpropanoid pathway are conducive to the establishment of a sensory transcription regulatory network associated with the $V v M Y B 44 \mathrm{TF}$ in BABA-primed grape resistance against B. cinerea.

In conclusion, VvMYB44, an R2R3-type MYB protein isolated from $V$. vinifera, was characterized in the BABA-primed defense response to the gray mold fungus $B$. cinerea. In the priming defense mode elicited by $10 \mathrm{mM}$ BABA, it was found that highly expressed VvMYB44 not only directly interacted with NPR1 in vivo but also activated the transcripts of enzymeencoding genes involved in sucrose metabolism and phenylpropanoid metabolism pathways, as demonstrated by taking both phytoalexin production and soluble sugar accumulation into account under the premise that the same level of resistance was achieved as in the direct defense mode furnished by $100 \mathrm{mM}$ BABA. Hence, $V v M Y B 44$ serves as a positive regulator of grape resistance to $B$. cinerea infection in an NPR1-dependent manner.
Moreover, the current study provides novel insight into the characteristics and functional roles of MYB TFs in activating the SA dependent signaling pathway.

\section{MATERIALS AND METHODS}

\section{Plant and fungus.}

Commercial mature grape berries $(V$. vinifera $\mathrm{L} . \times V$. labrusca L. 'Kyoho') with similar quality characteristics, as described by Li et al. (2021a), grown in a grape orchard following the standards of the Department of Agriculture (NY/T 3628-2020) were purchased and were hand-harvested in Tongnan District, Chongqing City, China, and were transported to our lab within $2 \mathrm{~h}$ of harvest.

Tobacco (Nicotiana tabacum) employed for the Co-IP assay was cultivated in an illuminated incubator (SPT-P500B, Darth Carter Co., Hefei, China) under a long-day (LD) photoperiod (60 to $70 \%$ relative humidity, 2,500 to 3,000 lux, $22^{\circ} \mathrm{C}$ under 14-h light and 10-h dark cycles) for at least 8 weeks.

The Col-0 ecotype of Arabidopsis thaliana was the genetic background for the transgenic plants presented in our current work. Col-0 WT seeds were surface-sterilized using sodium hypochlorite $(\mathrm{NaHClO})$ and were cold-stratified for approximately 3 days at $4^{\circ} \mathrm{C}$ before being sown in soil and cultured in the bicameral chamber system under a LD photoperiod for more than 4 weeks.

The B05.10 strain of Botrytis cinerea (Pers.: Fr.), isolated from decayed grapes (Lovato et al. 2019) and subsequently sequenced (Van Kan et al. 2017), was grown on potato dextrose agar medium (Solarbio, Beijing, China) at $26^{\circ} \mathrm{C}$ for 2 weeks until fungal sporulation. Conidia were obtained from sporulating cultures in $0.5 \%$ ( $\mathrm{vol} / \mathrm{vol})$ sterile water-diluted Tween-80. Then, the concentration of the spore suspensions was adjusted to $1.0 \times$ $10^{5}$ spores per milliliter, as assessed with a hemocytometer counting chamber.

\section{Grape treatments.}

Grapes were selected and were divided into three groups, each including 360 berries. We surface-sterilized these grapes with $75 \%$ (vol $/ \mathrm{vol})$ alcohol and arranged them on stainless steel mesh at $20^{\circ} \mathrm{C}$ for $1 \mathrm{~h}$. Thereafter, the berries from each group were immersed in BABA (purity $\geq 99 \%$ ) (Sigma, St. Louis) solutions containing $0.01 \%$ ( $\mathrm{vol} / \mathrm{vol}$ ) Tween-20 at concentrations of 0 (control), 10 , and $100 \mathrm{mM}$ at $20^{\circ} \mathrm{C}$ for $10 \mathrm{~min}$ and were dried in air for $3 \mathrm{~h}$. The two specific BABA concentrations were chosen based on our previous studies showing that 100 to $500 \mathrm{mM}$ BABA could induce a direct defense response and 10 to $50 \mathrm{mM}$ BABA activated a priming response (Wang et al. 2016, 2019). Next, the grapes were inoculated by spraying them with the prepared $B$. cinerea spore suspensions. After treatment, the berries were air-dried at $20^{\circ} \mathrm{C}$ for $3 \mathrm{~h}$, were sealed in polyethylene boxes $(10 \times 20 \mathrm{~cm}, 60 \mu \mathrm{m}$ thick $)$ and were incubated at $20 \pm$ $1{ }^{\circ} \mathrm{C}$ under 80 to $90 \%$ relative humidity for 5 days. Tissues of uninfected pulp from each replicate were collected at 1-day intervals from 0 to 5 days. Fruit pulp samples from each individual were flash-frozen in liquid $\mathrm{N}_{2}$ and were stored at $-80^{\circ} \mathrm{C}$ for analysis. This experiment was repeated two times in a completely randomized design (CRD) with three treatments and three replicates for each treatment.

\section{Assessment of disease development.}

A grape berry showing visible mold growth was regarded as decayed. The decay DI in grapes from the three BABA treatment groups subjected to B. cinerea spraying was estimated visually and was calculated at 1-day intervals. Based on the severity of the decay symptoms, the berries derived from the 
treatments with three concentrations of BABA were grouped into six grades ( $\mathrm{Li}$ et al. 2021b). The DI was calculated as described by Santana et al. (2011) and was expressed as the percentage of the total number of decayed grapes at each grade to the total number of berries.

\section{Determination of respiration rate, ET production, and MDA content.}

Berries $(1 \mathrm{~kg})$ from each treatment were sealed in 2-liter glass jars and were incubated at $20^{\circ} \mathrm{C}$ for $1.5 \mathrm{~h}$ for determination of $\mathrm{CO}_{2}$ production and $3.5 \mathrm{~h}$ for ET. Headspace gases were collected and were analyzed by a gas chromatograph equipped with a thermal conductivity detector for $\mathrm{CO}_{2}$ detection and a flame ionization detector for ET detection (McCollum and McDonald 1991). The respiration rate and ET production were expressed as milliliters of $\mathrm{CO}_{2}$ pre kilogram per hour and micoliters of ET per kilogram per hour, respectively.

Five grams of frozen tissues were extracted with $20 \mathrm{ml}$ of trichloroacetic acid and were centrifuged $(10,000 \times g, 5 \mathrm{~min}$, $4^{\circ} \mathrm{C}$ ). Then the collected supernatant was used to assess the MDA content by an improved thiobarbituric acid reaction system (Hodges et al. 1999). The results were presented as micromoles per kilogram of fresh weight $(\mathrm{FW})$.

\section{Determination of $\mathrm{H}_{2} \mathrm{O}_{2}$ content.}

One gram of frozen tissues was extracted with pure acetone $(10 \mathrm{ml})$ and was centrifuged $\left(12,000 \times g, 10 \mathrm{~min}, 4^{\circ} \mathrm{C}\right)$. The supernatant was applied to determine the endogenous $\mathrm{H}_{2} \mathrm{O}_{2}$ content by the titanium (IV) method (Patterson et al. 1984). The results were presented as micromoles per kilogram of FW.

\section{Determination of SA content.}

Five grams of frozen tissues were extracted twice with methanol $(25 \mathrm{ml})$ and were centrifuged $\left(12,000 \times g, 20 \mathrm{~min}, 4^{\circ} \mathrm{C}\right)$. The supernatant was concentrated to $2 \mathrm{ml}$ by rotary evaporation and were further dissolved with cold acetone overnight. This extract was subjected to SA analysis following the method of Meuwly and Métraux (1993).

\section{Determination of soluble sugars and UDPG contents.}

Tissue aliquots $(8 \mathrm{~g})$ were ground under liquid $\mathrm{N}_{2}$ and were extracted three times, each time with $40 \mathrm{ml}$ of $95 \%$ ( $\mathrm{vol} / \mathrm{vol}$ ) precooled ethanol. Then, the extract was centrifuged at $10,000 \times g$ for $15 \mathrm{~min}\left(4^{\circ} \mathrm{C}\right)$. The supernatant was collected and concentrated under vacuum $\left(37^{\circ} \mathrm{C}\right)$, using a rotary evaporator. Furthermore, the residue was dissolved in deionized water, and the high-performance liquid chromatography (HPLC) methods of Wilson et al. (1981) and Goulard et al. (2001) were employed to quantify the soluble sugar and UDPG contents in grape extracts, respectively.

\section{Determination of phytoalexin contents.}

To obtain extracts of phytoalexins, $5 \mathrm{~g}$ of ground fruit tissue was homogenized in $30 \mathrm{ml}$ of precooled methanol $(85 \%$, vol/ vol). Thereafter, the homogenate was kept in the dark overnight $\left(4^{\circ} \mathrm{C}\right)$ and was then centrifuged $(10,000 \times g, 15 \mathrm{~min})$. The supernatant was evaporated under nitrogen at $20^{\circ} \mathrm{C}$, and the residue was dissolved in methanol and was filtrated by nylon fiber niters. The contents of phytoalexins, including trans-resveratrol and $\varepsilon$-viniferin, in the prepared extracts were measured by the HPLC method described by Vitrac et al. (2005).

\section{RNA isolation and real-time qPCR.}

Total RNA was extracted from the powdered frozen samples (5 g) with the RNAprep Pure Kit for polysaccharide- and polyphenolic-rich plant (Tiangen, Beijing, China), and singlestranded complementary DNA (cDNA) was synthesized from aliquots $(1 \mu \mathrm{g})$ of RNA using a PrimeScript RT reagent kit
(Takara, Tokyo) (Cao et al. 2021; Wang et al. 2020). Moreover, qPCR was conducted with SYBR Green in triplicate on an Applied Biosystems (ABI) 7500 FAST real-time PCR system. Gene-specific primer pairs (listed in Supplementary Table S1) were designed via Primer3 software, available in the National Center for Biotechnology Information (NCBI) database, with expected amplicon lengths between 90 and $150 \mathrm{bp}$, to minimize the impact of RNA and ensure optimal polymerization efficiency. qPCRs were performed in a $20-\mu \mathrm{l}$ reaction volume (final oligonucleotide concentration of $200 \mathrm{nM}$ of each primer and $2 \mu \mathrm{l}$ of cDNA template) with $10 \mu \mathrm{l}$ of SYBR qPCR Master Mix (Vazyme, Nanjing, China) on an ABI 7500 instrument using the following thermal cycling program: $95^{\circ} \mathrm{C}$ for $30 \mathrm{~s}$, followed by 40 cycles of $95^{\circ} \mathrm{C}$ for $10 \mathrm{~s}$ and $60^{\circ} \mathrm{C}$ for $30 \mathrm{~s}$, followed by melt curve analysis consisting of $95^{\circ} \mathrm{C}$ for $15 \mathrm{~s}, 60^{\circ} \mathrm{C}$ for $60 \mathrm{~s}$, and $95^{\circ} \mathrm{C}$ for $15 \mathrm{~s}$. The qPCR results were normalized following the $2^{-\Delta \Delta C T}$ method (Livak and Schmittgen 2001). In this case, the reference gene Vv18S rRNA (GenBank AF321266.1) was taken as the internal control of $V$. vinifera because the gene was consistently expressed in grape samples over the storage period (Wang et al. 2019). In addition, Actin2 was used as the reference gene of Arabidopsis. Moreover, the gene expression levels were normalized based on the value for the control plant, which was set to 1 .

\section{RNA preparation, Illumina sequencing and data preprocessing.}

Given that treatment of grapes with both 10 and $100 \mathrm{mM}$ BABA induced potentiated resistance performances within a day after treatments, as reflected in the obviously increased transcripts of $P R$ genes (Fig. 3C), we analyzed the transcriptomic responses of grapes treated with different BABA concentrations on the first day. Total RNA from grape pulp was isolated as mentioned above (RNA isolation and real-time qPCR), and the cDNA synthesized from the enriched mRNAs was sequenced based on Illumina HiSeq 2500 paired-end sequencing technology. The obtained raw sequence data have been uploaded to the NCBI sequence read archive under the BioProject accession (PRJNA509494). Sequenced reads were further demultiplexed, filtered, and mapped to the grapevine genome; in addition, the unique mapped reads were subjected to transcriptome reconstruction using Trinity software (v.2.2.0), and read counts were calculated and normalized as described by Wang et al. (2020).

\section{Transcriptome comparison and functional annotation.}

Those assembled transcripts were further filtered to retrieve the DEGs with $\log _{2} \mathrm{FC}$ values greater than $1(\mid P$ value $\leq 0.05$ and false discovery rate $<0.05$ in $10 \mathrm{mM}$ BABA-vs-control, 100 $\mathrm{mM}$ BABA-vs-control, and $10 \mathrm{mM}$ BABA-vs-100 mM BABA pairwise comparisons using the edgeR package (Robinson et al. 2010). A Venn diagram was employed to identify the coexpressed DEGs among the above three pairwise comparisons. Moreover, Blast2GO was utilized to obtain the GO classifications of DEGs on the basis of the biological process, cellular component and molecular function ontologies (Conesa et al. 2005). DEGs were further aligned to the KOG and KEGG databases to identify their functional distribution characteristics and their differences in biological functions (Schuler et al. 2011).

\section{qPCR validation.}

qPCR analysis of all critical regulatory DEGs in the grapevine transcriptome involved in the phenylpropanoid pathway and sucrose metabolism was carried out to verify their expression in response to BABA-elicited defense responses. The RNA extraction, cDNA synthesis, and qPCR analysis procedures are described above, and the specific primers used for DEGs are listed in Supplementary Table S1. 
Identification, phylogenetic analysis, and functional prediction of MYB TFs in $V$. vinifera.

Since the positive function of the R2R3-type MYB44 protein in Arabidopsis defense against fungal pathogens, mainly through the SA signaling pathway, has been characterized (Shim et al. 2013; Zou et al. 2013) but a potentially similar MYB defense factor from $V$. vinifera remains to be identified, we performed a combined phylogenetic analysis of AtMYB44 and $V$. vinifera MYBs using the neighbor-joining method to characterize the evolutionary relationships of $V v M Y B s$ and identify potential orthologs of AtMYB44 from $V$. vinifera. Two approaches to identify members of the $V$. vinifera MYB gene family were applied. On the one hand, we determined MYB and V. vinifera as keywords to search the NCBI database and downloaded all of the retrieved sequences. Furthermore, we set the amino acid sequence of AtMYB44 as the "seed" sequence and conducted a BLASTp search of AtMYB44 against the nonredundant protein sequences of $V$. vinifera and selected all of the nonhypothetical sequences. The domain composition of all the downloaded protein sequences was examined using the SMART domain analysis program with default parameters. Then, we weeded out redundant proteins and successfully obtained $50 V v M Y B$ genes. The phylogenetic tree was constructed on the basis of multiple sequence alignment in MEGA 5.0 with 1,000 bootstrap replicates. The phylogenetic tree was further prettified by the online tool iTOL (Letunic and Bork 2007). Additionally, the biological functions of certain $V$. vinifera MYBs were predicted according to orthologous relationships derived from the aforementioned phylogenetic tree.

\section{Subcellular localization of $V v M Y B 44$.}

The coding region of the identified $V v M Y B 44$ sequence was introduced into the binary expression vector pCAMBIA130135S-GFP at the $5^{\prime}$ terminus of GFP, driven by the cauliflower mosaic virus $35 \mathrm{~S}$ promoter. Afterward, onion (Allium cepa) bulb scale epidermis cells were transformed by inoculation with Agrobacterium tumefaciens EHA105 harboring the empty pEAQ-GFP vector and the pEAQ-VvMYB44-GFP recombinant protein as described by Li et al. (2021a). Following 45 to $70 \mathrm{~h}$ of cultivation on Murashige and Skoog (MS) medium in darkness $\left(26^{\circ} \mathrm{C}\right)$, the epidermal cells were stained with DAPI (Sigma) at $20 \mu \mathrm{g} \mathrm{ml}^{-1}$. The fluorescence signals of the stained onion peels were visualized by fluorescence microscopy (Zeiss LSM 510) with excitation wavelengths at 488 and $405 \mathrm{~nm}$ for GFP and DAPI fluorescence, respectively, at 3 to $6 \mathrm{~h}$ after staining. Here, pEAQ-GFP served as the positive control.

\section{Y2H assay.}

$\mathrm{Y} 2 \mathrm{H}$ analysis was further carried out by employing the Matchmaker GAL4-based Y2H system (Clontech, Mountain View, CA, U.S.A.) to verify the interaction between $V v M Y B 44$ and $V v N P R 1$. The coding regions of $V v M Y B 44$ or $V v N P R 1$ were introduced into the pGBKT7 (BA) or pGADT7 (AD) plasmid to construct the bait (BD-VvMYB44) or prey (AD-VvNPR1) expression vector, respectively. The two constructs were fused to yeast AH109 cells, which were then cultured on synthetic dropout media with the addition of $\mathrm{X}-\boldsymbol{\alpha}$-gal ( $40 \mathrm{mg}$ per liter) as previously described (Wang et al. 2021) to test for possible interaction.

\section{Co-IP assay.}

A Co-IP assay was performed as Wang et al. (2021) described. In detail, the full-length coding sequences (CDSs) of $V v M Y B 44$ and $V v N P R 1$ were introduced into pBinGFP2 and pCAMBIA35s-4 $\times$ myc, respectively. The recombinant proteins (VvMYB44-GFP harboring a GFP-labeled antibody and VvNPRICAM harboring a myc-labeled antibody) were transformed into
A. tumefaciens GV3101 cells, which were then infiltrated into 8 -week-old tobacco leaves. The infected leaves were cultivated for an additional $38 \mathrm{~h}$ under LD conditions. The isolated leaf protein was either directly subjected to $12 \%$ sodium dodecyl sulfatepolyacrylamide gel electrophoresis (SDS-PAGE) for Western immunoblotting analysis or immunoprecipitated with a horseradish peroxidase (HRP)-conjugated anti-GFP antibody (Invitrogen, Carlsbad, CA, U.S.A.) (1:2,000) or a HRP-conjugated anti-myc antibody (Invitrogen) (1:2,000). The immune complexes were collected using protein A/G-agarose (Santa Cruz Biotechnology, Santa Cruz, CA, U.S.A.) and were washed eight times with commercial wash buffer. For immunoblotting, the isolated protein or pellet was suspended in $1 \times$ SDS-PAGE loading buffer, was separated on SDS-PAGE gels, and was transferred to a nitrocellulose membrane. The membrane was immunoblotted with anti-GFP or anti-myc antibody and was visualized with an ECL-Plus Western blotting system (GE Healthcare, Piscataway, NJ, U.S.A.).

\section{Y1H assay.}

The Y1H assay was conducted under the Matchmaker gold $\mathrm{Y} 1 \mathrm{H}$ system (manufactured by Clontech). Three copies of the MBS I [CNGTT(A/G)] or MBS II [C(G/T)T(A/T)GTT(A/G)]motif cis-acting elements and their adjacent nucleotides (approximately $50 \mathrm{bp}$ ) were synthesized, based on the promoter fragments of sucrose and phenylpropanoid metabolism-related genes (presented in Supplementary Data S2), and were ligated into the pAbAi vector, and then $V v S S 2-\mathrm{AbAi}, V v S P S 3-\mathrm{AbAi}, V v S P P 1-$ AbAi, VvUDPase3-AbAi, VvAI-AbAi, VvNI-AbAi, $V v P A L-$ AbAi, Vv4CL-AbAi, VvC4H-AbAi, VvCYP73A-AbAi, VvSTS1$\mathrm{AbAi}$, and $\mathrm{p} 53-\mathrm{AbAi}$ were linearized by BbsI and transformed into the Y1HGold strain. The construction of recombinant $V v M Y B 44-p G A D T 7$, the determination of the minimal inhibitory concentration of aureobasidin $\mathrm{A}(\mathrm{AbA})$ and the detection of direct binding among $V v M Y B 44, V v S S 2, V v S P S 3, V v S P P 1, V v U D P a s e 3$, $V v A I, V v N I, V v P A L, V v 4 C L, V v C 4 H, V v C Y P 73 A$, and $V v S T S 1$ were performed according to our previous study (Wang et al. 2021).

\section{DLR assay.}

The transactivation of the promoters of enzymes related to the phenylpropanoid pathway and sucrose metabolism by $V v M Y B 44$ was determined with a dual-LUC system. In detail, the CDS fragment of $V v M Y B 44$ from $10 \mathrm{mM}$ BABA-treated grapes was introduced into the pGreenII 62-SK vector to serve as the effecter (35S::VvMYB44). The promoters of the downstream target genes of $V v M Y B 44$, including genes encoding enzymes involved in sucrose metabolism (SS2, SPS3, SPP1, UDPase3, $\mathrm{AI}$, and $\mathrm{NI}$ ) and phenylpropanoid pathway (PAL, 4CL, C4H, CYP73A, and STS1), were inserted into the pGreenII 0800LUC vector to construct reporters (Pro SS2/SPS3/SPPI/UDPase3/AI/NII $P A L / 4 C L / C 4 H / C Y P 73 A / S T S 1: \because L U C)$. The effecter and reporters were then fused to A. tumefaciens GV3101. The GV3101 cells harboring $35 S:: V v M Y B 44$ were blended with each reporter in equal proportions and were infiltrated into 5- to 9-week-old tobacco plants (abaxial leaf surfaces). The binding activities of $V v M Y B 44$ to the related promoters were determined as recorded by Shan et al. (2016) and reported as the LUC/REN ratio.

Transgenic Arabidopsis overexpressing $V$. vinifera MYB44. The amplified CDS fragment of VvMYB44 was fused into the SacI and BamHI restriction sites of binary vector pCAMBIA 1305.1 to obtain the heterologous overexpression plasmid 35S::VvMYB44. Arabidopsis plants were infected with GV3101 harboring 35S::VvMYB44 via floral dip method (Clough and Bent 1998). After surface-sterilizing by immersion in $2 \% \mathrm{NaH}-$ $\mathrm{ClO}$ for $10 \mathrm{~min}$, the harvested $\mathrm{T} 1$ (transgenic generation 1) seeds were stratified in double-distilled $\mathrm{H}_{2} \mathrm{O}$ for 3 days at $4^{\circ} \mathrm{C}$ followed 
by seeding on half-strength MS basal medium with the addition of kanamycin (50 mg per liter). The kanamycin-resistant seedlings were grown in pots containing perlite and vermiculite $(1: 2$, $\mathrm{vol} / \mathrm{vol}$ ) under LD conditions. In the T3 generation, 15 independent homozygous transgenic lines were obtained, which were selected for further analysis.

\section{Inoculation of Arabidopsis with $B$. cinerea after drenching with a BABA formulation.}

After drench treatment with $30 \mu \mathrm{g}$ of BABA per milliliter, following the previously reported method of Zimmerli et al. (2001), the soil-grown T3 generation of transgenic Arabidopsis and WT Col-0 plants were dot-inoculated with $10-\mu \mathrm{l}$ droplets of a $B$. cinerea conidial suspension $\left(1.0 \times 10^{5}\right.$ conidia per milliliter $)$ at fixed positions to the left and right of the midvein, as described by Xiao and Chye (2011). Leaf samples were selected for either phenotype observations or gene expression determinations at one or both 3 and 6 dpi.

\section{Trypan blue staining.}

The B. cinerea-infiltrated T3 generation of transgenic Arabidopsis and WT plant leaves were boiled in $0.025 \%$ aqueous trypan blue solution as described in our current study ( $\mathrm{Li}$ et al. 2021a). After being immersed continuously in saturated chloral hydrate for at least $20 \mathrm{~h}$, the trypan blue-stained leaves were photographed by a binocular stereo-microscope (Leica EZ4D).

\section{Data analysis.}

Data analyses were performed using the Data Processing System. The results are indicated as the means \pm standard error of independent biological replicates and were analyzed by one-way analysis of variance. Differences in significance were analyzed using Duncan-type multiple comparisons, and differences showing $P<0.05$ were considered significant.

\section{ACKNOWLEDGMENTS}

We specially thank Y. Liao, S. Chen, and D. Wu for sample preparation during the past five years.

\section{LITERATURE CITED}

Agarwal, M., Hao, Y., Kapoor, A., Dong, C. H., Fujii, H., Zheng, X., and Zhu, J. K. 2006. A R2R3 type MYB transcription factor is involved in the cold regulation of $\mathrm{CBF}$ genes and in acquired freezing tolerance. J. Biol. Chem. 281:37636-37645.

Bomal, C., Duval, I., Giguère, I., Fortin, É., Caron, S., Stewart, D., Boyle, B., Séguin, A., and MacKay, J. J. 2014. Opposite action of R2R3-MYBs from different subgroups on key genes of the shikimate and monolignol pathways in spruce. J. Exp. Bot. 65:495-508.

Borsani, J., Budde, C. O., Porrini, L., Lauxmann, M. A., Lombardo, V. A., Murray, R., Andreo, C. S., Drincovich, M. F., and Lara, M. V. 2009. Carbon metabolism of peach fruit after harvest: Changes in enzymes involved in organic acid and sugar level modifications. J. Exp. Bot. 60:1823-1837.

Cao, Y., Xu, L., Xu, H., Yang, P., He, G., Tang, Y., Qi, X., Song, M. and Ming, J. 2021. LhGST is an anthocyanin-related glutathione S-transferase gene in Asiatic hybrid lilies (Lilium spp.). Plant Cell Rep. 40:85-95.

Cheng, H., Song, S., Xiao, L., Soo, H. M., Cheng, Z., Xie, D., and Peng, J. 2009. Gibberellin acts through jasmonate to control the expression of MYB21, MYB24, and MYB57 to promote stamen filament growth in Arabidopsis. PLoS Genet. 5:e1000440-e1000453.

Chezem, W. R., Memon, A., Li, F. S., Weng, J. K., and Clay, N. K. 2017. SG2-type R2R3-MYB transcription factor MYB15 controls defense-induced lignification and basal immunity in Arabidopsis. Plant Cell 29:1907-1926.

Ciereszko, I., Johansson, H., and Kleczkowski, L. A. 2001. Sucrose and light regulation of a cold-inducible UDP-glucose pyrophosphorylase gene via a hexokinase-independent and abscisic acid-insensitive pathway in Arabidopsis. Biochem. J. 354:67-72.
Clough, S. J., and Bent, A. F. 1998. Floral dip: A simplified method for Agrobacterium-mediated transformation of Arabidopsis thaliana. Plant J. 16:735-743.

Cohen, Y., Vaknin, M., and Mauch-Mani, B. 2016. BABA-induced resistance: Milestones along a 55-year journey. Phytoparasitica 44:513-538.

Conesa, A., Götz, S., García-Gómez, J. M., Terol, J., Talón, M., and Robles, M. 2005. Blast2GO: A universal tool for annotation, visualization and analysis in functional genomics research. Bioinformatics 21: 3674-3676.

Conrath, U., Beckers, G. J. M., Flors, V., García-Agustín, P., Jakab, G., Mauch, F., Newman, M. A., Pieterse, C. M. J., Poinssot, B., Pozo, M. J., Pugin, A., Schaffrath, U., Ton, J., Wendehenne, D., Zimmerli, L., and Mauch-Mani, B. 2006. Priming: Getting ready for battle. Mol. Plant-Microbe Interact. 19:1062-1071.

Dai, Z. W., Ollat, N., Gomes, E., Decroocq, S., Tandonnet, J. P., Bordenave, L., Pieri, P., Hilbert, G., Kappel, C., van Leeuwen, C. Vivin, P., and Delrot, S. 2011. Ecophysiological, genetic, and molecular causes of variation in grape berry weight and composition: A review. Am. J. Enol. Vitic. 62:413-425.

de Leone, M. J., Hernando, C. E., Mora-García, S., and Yanovsky, M. J. 2020. It's a matter of time: The role of transcriptional regulation in the circadian clock-pathogen crosstalk in plants. Transcription 11:100-116.

Du, H., Wang, Y. B., Xie, Y., Liang, Z., Jiang, S. J., Zhang, S. S., Huang, Y. B., and Tang, Y. X. 2013. Genome-wide identification and evolutionary and expression analyses of MYB-related genes in land plants. DNA Res. 20:437-448.

Dubos, C., Stracke, R., Grotewold, E., Weisshaar, B., Martin, C., and Lepiniec, L. 2010. MYB transcription factors in Arabidopsis. Trends Plant Sci. 15:573-581.

Dubreuil-Maurizi, C., Trouvelot, S., Frettinger, P., Pugin, A., Wendehenne, D., and Poinssot, B. 2010. $\beta$-aminobutyric acid primes an NADPH oxidase-dependent reactive oxygen species production during grapevinetriggered immunity. Mol. Plant-Microbe Interact. 23:1012-1021.

Feliziani, E., Smilanick, J. L., Margosan, D. A., Mansour, M. F., Romanazzi, G., Gu, S., Gohil, H. L., and Ames, Z. R. 2013. Preharvest fungicide, potassium sorbate, or chitosan use on quality and storage decay of table grapes. Plant Dis. 97:307-314.

Fornalé, S., Sonbol, F. M., Maes, T., Capellades, M., Puigdomènech, P., Rigau, J., and Caparrós-Ruiz, D. 2006. Down-regulation of the maize and Arabidopsis thaliana caffeic acid $O$-methyl-transferase genes by two new maize R2R3-MYB transcription factors. Plant Mol. Biol. 62: 809-823.

Fu, Y. H., Wei, J. J., Pan, Y. B., Zhou, V., He, E. Q., Liu, R., Huang, H., Lu, J. J., and Liu, F. Z. 2019. Comparative analysis reveals changes in transcriptomes of sugarcane upon infection by Leifsoniaxyli subsp. xyli. J. Phytopathol. 167:633-644.

Gonzalez, A., Zhao, M., Leavitt, J. M., and Lloyd, A. M. 2008. Regulation of the anthocyanin biosynthetic pathway by the TTG1/bHLH/Myb transcriptional complex in Arabidopsis seedlings. Plant J. 53:814-827.

Goulard, F., Diouris, M., Deslandes, E., and Floc'h, J. Y. 2001. An HPLC method for the assay of UDP-glucose pyrophosphorylase and UDP-glucose-4-epimerase in Solieria chordalis (Rhodophyceae). Phytochem. Anal. 12:363-365

Hieno, A., Naznin, H. A., Hyakumachi, M., Higuchi-Takeuchi, M., Matsui, M., and Yamamoto, Y. Y. 2016. Possible involvement of MYB44-mediated stomatal regulation in systemic resistance induced by Penicillium simplicissimum GP17-2 in Arabidopsis. Microbes Environ. 31:154-159.

Hill, C. S., and Treisman, R. 1995. Transcriptional regulation by extracellular signals: Mechanisms and specificity. Cell 80:199-211.

Hodges, D. M., DeLong, J. M., Forney, C. F., and Prange, R. K. 1999. Improving the thiobarbituric acid-reactive-substances assay for estimating lipid peroxidation in plant tissues containing anthocyanin and other interfering compounds. Planta 207:604-611.

Jung, C., Seo, J. S., Han, S. W., Koo, Y. J., Kim, C. H., Song, S. I., Nahm, B. H., Choi, Y. D., and Cheong, J. J. 2008. Overexpression of AtMYB44 enhances stomatal closure to confer abiotic stress tolerance in transgenic Arabidopsis. Plant Physiol. 146:323-324.

Jung, C., Shim, J. S., Seo, J. S., Lee, H. Y., Kim, C. H., Choi, Y. D., and Cheong, J. J. 2010. Non-specific phytohormonal induction of AtMYB44 and suppression of jasmonate-responsive gene activation in Arabidopsis thaliana. Mol. Cells 29:71-76.

Jung, H. W., Tschaplinski, T. J., Wang, L., Glazebrook, J., and Greenberg, J. T. 2009. Priming in systemic plant immunity. Science 324:89-91.

Katiyar, A., Smita, S., Lenka, S. K., Rajwanshi, R., Chinnusamy, V., and Bansal, K. C. 2012. Genome-wide classification and expression analysis of $M Y B$ transcription factor families in rice and Arabidopsis. BMC Genomics 13:544-562. 
Khare, S., Singh, N. B., Singh, A., Hussain, I., Niharika, K., Yadav, V., Bano, C., Yadav, R., and Amist, N. 2020. Plant secondary metabolites synthesis and their regulations under biotic and abiotic constraints. J. Plant Biol. 11:1-14

Kim, S., Kim, C. Y., Park, S. Y., Kim, K. T., Jeon, J., Chung, H., Choi, G., Kwon, S., Choi, J., Jeon, J., Jeon, J. S., Khang, C. H., Kang, S., and Lee, Y. H. 2020. Two nuclear effectors of the rice blast fungus modulate host immunity via transcriptional reprogramming. Nat. Commun. 11:5845-5856.

Kleczkowski, L. A. 1994. Glucose activation and metabolism through UDPglucose pyrophosphorylase in plants. Phytochemistry 37:1507-1515.

Letunic, I., and Bork, P. 2007. Interactive Tree Of Life (iTOL): An online tool for phylogenetic tree display and annotation. Bioinformatics 23:127-128.

Levine, A., Tenhaken, R., Dixon, R., and Lamb, C. 1994. $\mathrm{H}_{2} \mathrm{O}_{2}$ from the oxidative burst orchestrates the plant hypersensitive disease resistance response. Cell 79:583-593.

Li, C., Cao, S., Wang, K., Lei, C., Ji, N., Xu, F., Jiang, Y., Qiu, L., and Zheng, Y. 2021a. Heat shock protein HSP24 is involved in the BABA-induced resistance to fungal pathogen in postharvest grapes underlying an NPR1-dependent manner. Front. Plant Sci. 12:646147646160

Li, C. H., Wang, J., Ji, N. N., Lei, C. Y., Zhou, D. X., Zheng, Y. H., and Wang, K. T. 2020. PpHOS1, a RING E3 ubiquitin ligase, interacts with $P$ PWRKY22 in the BABA-induced priming defense of peach fruit against Rhizopus stolonifer. Postharvest Biol. Technol. 159:111029111037.

Li, C. H., Wang, K. T., Xu, F., Lei, C. Y., Jiang, Y. B., and Zheng, Y. H. 2021b. Sucrose metabolism and sensory evaluation in peach as influenced by $\beta$-aminobutyric acid (BABA)-induced disease resistance and the transcriptional mechanism involved. Postharvest Biol. Technol. 174:111465-111476.

Li, X., Wang, C., Cheng, J., Zhang, J., da Silva, J. A. T., Liu, X., Duan, X., Li, T., and Sun, H. 2014. Transcriptome analysis of carbohydrate metabolism during bulblet formation and development in Lilium davidii var. unicolor. BMC Plant Biol. 14:358-369.

Liu, R., Lü, B., Wang, X., Zhang, C., Zhang, S., Qian, J., Chen, L., Shi, H., and Dong, H. 2010. Thirty-seven transcription factor genes differentially respond to a harpin protein and affect resistance to the green peach aphid in Arabidopsis. J. Biosci. 35:435-450.

Livak, K. J., and Schmittgen, T. D. 2001. Analysis of relative gene expression data using real-time quantitative PCR and the $2^{-\Delta \Delta C(T)}$ method. Methods 25:402-408.

Lovato, A., Zenoni, S., Tornielli, G. B., Colombo, T., Vandelle, E., and Polverari, A. 2019. Specific molecular interactions between Vitis vinifera and Botrytis cinerea are required for noble rot development in grape berries. Postharvest Biol. Technol. 156:110924-110938.

Luna, E., Van Hulten, M., Zhang, Y., Berkowitz, O., López, A., Pétriacq, P., Sellwood, M. A., Chen, B., Burrell, M., van de Meene, A., Pieterse, C. M. J., Flors, V., and Ton, J. 2014. Plant perception of $\beta$-aminobutyric acid is mediated by an aspartyl-tRNA synthetase[J]. Nat. Chem. Biol. 10:450-456.

Lü, B., Sun, W., Zhang, S., Zhang, C., Qian, J., Wang, X., Gao, R., and Dong, H. 2011. HrpN Ea-induced deterrent effect on phloem feeding of the green peach aphid Myzus persicae requires AtGSL5 and AtMYB44 genes in Arabidopsis thaliana. J. Biosci. 36:123-137.

Lyons, R., Stiller, J., Powell, J., Rusu, A., Manners, J. M., and Kazan, K. 2015. Fusarium oxysporum triggers tissue-specific transcriptional reprogramming in Arabidopsis thaliana. PLoS One 10:e121902e0121925.

Ma, D., Reichelt, M., Yoshida, K., Gershenzon, J., and Constabel, C. P. 2018. Two R2R3-MYB proteins are broad repressors of flavonoid and phenylpropanoid metabolism in poplar. Plant J. 96:949-965.

Martin, C., and Paz-Ares, J. 1997. MYB transcription factors in plants. Trends Genet. 13:67-73.

Mazid, M., Khan, T. A., and Mohammad, F. 2011. Role of nitric oxide in regulation of $\mathrm{H}_{2} \mathrm{O}_{2}$ mediating tolerance of plants to abiotic stress: A synergistic signaling approach. J. Physiol. Biochem. 7:34-74.

McCollum, T. G., and McDonald, R. E. 1991. Electrolyte leakage, respiration, and ethylene as indices of chilling injury in grapefruit. HortScience 26:1191-1192

Meissner, R. C., Jin, H., Cominelli, E., Denekamp, M., Fuertes, A., Greco, R., Kranz, H. D., Penfield, S., Petroni, K., Urzainqui, A., Martin, C., Paz-Ares, J., Smeekens, S., Tonelli, C., Weisshaar, B., Baumann, E., Klimyuk, V., Marillonnet, S., Patel, K., Speulman, E., Tissier, A. F., Bouchez, D., Jones, J. J., Pereira, A., Wisman, E., and Bevan, M. 1999. Function search in a large transcription factor gene family in Arabidopsis: Assessing the potential of reverse genetics to identify insertional mutations in R2R3 $M Y B$ genes. Plant Cell 11: 1827-1840

Mengiste, T., Chen, X., Salmeron, J., and Dietrich, R. 2003. The BOTRYTIS SUSCEPTIBLE1 gene encodes an R2R3MYB transcription factor protein that is required for biotic and abiotic stress responses in Arabidopsis. Plant Cell 15:2551-2565.

Meuwly, P., and Métraux, J. P. 1993. Ortho-anisic acid as internal standard for the simultaneous quantitation of salicylic acid and its putative biosynthetic precursors in cucumber leaves. Anal. Biochem. 214:500-505.

Miron, D., and Schaffer, A. A. 1991. Sucrose phosphate synthase sucrose synthase, and invertase activities in developing fruit of lycopersicon esculentum Mill. and the sucrose accumulating Lycopersicon hirsutum Humb. and Bonpl. Plant Physiol. 95:623-627.

Morkunas, I., Narożna, D., Nowak, W., Samardakiewicz, S., and RemleinStarosta, D. 2011. Cross-talk interactions of sucrose and Fusarium oxysporum in the phenylpropanoid pathway and the accumulation and localization of flavonoids in embryo axes of yellow lupine. J. Plant Physiol. 168:424-433.

Morkunas, I., and Ratajczak, L. 2014. The role of sugar signaling in plant defense responses against fungal pathogens. Acta Physiol. Plant. 36: 1607-1619.

Mostek, A., Börner, A., and Weidner, S. 2016. Comparative proteomic analysis of $\beta$-aminobutyric acid-mediated alleviation of salt stress in barley. Plant Physiol. Biochem. 99:150-161.

O'Brien, J. A., Daudi, A., Butt, V. S., and Bolwell, G. P. 2012. Reactive oxygen species and their role in plant defence and cell wall metabolism. Planta 236:765-779.

Panda, S., Busatto, N., Bhat, K., and Kamble, A. C. 2019. Piriformospora indica-primed transcriptional reprogramming induces defense response against early blight in tomato. Sci. Hortic. (Amsterdam) 255:209-219.

Patterson, B. D., MacRae, E. A., and Ferguson, I. B. 1984. Estimation of hydrogen peroxide in plant extracts using titanium(IV). Anal. Biochem. 139:487-492.

Peng, X., Liu, H., Wang, D., and Shen, S. 2016. Genome-wide identification of the Jatropha curcas MYB family and functional analysis of the abiotic stress responsive gene JcMYB2. BMC Genomics 17:251-263.

Persak, H., and Pitzschke, A. 2014. Dominant repression by Arabidopsis transcription factor MYB44 causes oxidative damage and hypersensitivity to abiotic stress. Int. J. Mol. Sci. 15:2517-2537.

Preston, J., Wheeler, J., Heazlewood, J., Li, S. F., and Parish, R. W. 2004. AtMYB32 is required for normal pollen development in Arabidopsis thaliana. Plant J. 40:979-995.

Qin, G., Zhu, Z., Wang, W., Cai, J., Chen, Y., Li, L., and Tian, S. 2016. A tomato vacuolar invertase inhibitor mediates sucrose metabolism and influences fruit ripening. Plant Physiol. 172:1596-1611.

Qiu, Z., Yan, S., Xia, B., Jiang, J., Yu, B., Lei, J., Chen, C., Chen, L., Yang, Y., Wang, Y., Tian, S., and Cao, B. 2019. The eggplant transcription factor MYB44 enhances resistance to bacterial wilt by activating the expression of spermidine synthase. J. Exp. Bot. 70:5343-5354.

Robinson, M. D., McCarthy, D. J., and Smyth, G. K. 2010. edgeR: A Bioconductor package for differential expression analysis of digital gene expression data. Bioinformatics 26:139-140.

Romero, I., Fuertes, A., Benito, M. J., Malpica, J. M., Leyva, A., and Paz-Ares, J. 1998. More than 80R2R3-MYB regulatory genes in the genome of Arabidopsis thaliana. Plant J. 14:273-284.

Sablowski, R. W. M., Moyano, E., Culianez-Macia, F. A., Schuch, W., Martin, C., and Bevan, M. 1994. A flower-specific Myb protein activates transcription of phenylpropanoid biosynthetic genes. EMBO J. 13:128-137.

Santana, L. R. R. D., Benedetti, B. C., Sigrist, J. M. M., Sato, H. H., and Anjos, V. D. D. A. 2011. Effect of controlled atmosphere on postharvest quality of 'Douradão' peaches.. Food Sci. Technol. 31:231-237.

Schirra, M., D’Aquino, S., Cabras, P., and Angioni, A. 2011. Control of postharvest diseases of fruit by heat and fungicides: Efficacy, residue levels, and residue persistence. A review. J. Agric. Food Chem. 59: 8531-8542.

Schuler, M., Keller, A., Backes, C., Philippar, K., Lenhof, H. P., and Bauer, P. 2011. Transcriptome analysis by GeneTrail revealed regulation of functional categories in response to alterations of iron homeostasis in Arabidopsis thaliana. BMC Plant Biol. 11:87.

Seo, J., Sohn, H., Noh, K., Jung, C., An, J., Donovan, C. M., Somers, D. A., Kim, D. I., Jeong, S. C., Kim, C. G., Kim, H. M., Lee, S. H., Choi, Y. D., Moon, T. W., Kim, C. H., and Cheong, J. J. 2012. Expression of the Arabidopsis AtMYB44 gene confers drought/saltstress tolerance in transgenic soybean. Mol. Breed. 29:601-608.

Seo, P. J., and Park, C. M. 2010. MYB96-mediated abscisic acid signals induce pathogen resistance response by promoting salicylic acid biosynthesis in Arabidopsis. New Phytol. 186:471-483. 
Shan, W., Chen, J. Y., Kuang, J. F., and Lu, W. J. 2016. Banana fruit NAC transcription factor MaNAC5 cooperates with MaWRKYs to enhance the expression of pathogenesis-related genes against Colletotrichum musae. Mol. Plant Pathol. 17:330-338.

Shim, J. S., Jung, C., Lee, S., Min, K., Lee, Y. W., Choi, Y., Lee, J. S., Song, J. T., Kim, J. K., and Choi, Y. D. 2013. AtMYB44 regulates WRKY70 expression and modulates antagonistic interaction between salicylic acid and jasmonic acid signaling. Plant J. 73:483-495.

Smith, J. E., Mengesha, B., Tang, H., Mengiste, T., and Bluhm, B. H. 2014. Resistance to Botrytis cinerea in Solanum lycopersicoides involves widespread transcriptional reprogramming. BMC Genomics 15:334-352.

Stracke, R., Ishihara, H., Huep, G., Barsch, A., Mehrtens, F., Niehaus, K., and Weisshaar, B. 2007. Differential regulation of closely related R2R3MYB transcription factors controls flavonol accumulation in different parts of the Arabidopsis thaliana seedling. Plant J. 50:660-677.

Stracke, R., Werber, M., and Weisshaar, B. 2001. The R2R3-MYB gene family in Arabidopsis thaliana. Curr. Opin. Plant Biol. 4:447-456.

Tamagnone, L., Merida, A., Parr, A., Mackay, S., Culianez-Macia, F. A., Roberts, K., and Martin, C. 1998. The AmMYB308 and AmMYB330 transcription factors from antirrhinum regulate phenylpropanoid and lignin biosynthesis in transgenic tobacco. Plant Cell 10:135-154.

Thevenet, D., Pastor, V., Baccelli, I., Balmer, A., Vallat, A., Neier, R., Glauser, G., and Mauch-Mani, B. 2017. The priming molecule $\beta$-aminobutyric acid is naturally present in plants and is induced by stress. New Phytol. 213:552-559.

Thom, M., Leigh, R. A., and Maretzki, A. 1986. Evidence for the involvement of a UDP-glucose-dependent group translocator in sucrose uptake into vacuoles of storage roots of red beet. Planta 167:410-413.

Ton, J., Jakab, G., Toquin, V., Flors, V., Iavicoli, A., Maeder, M. N., Métraux, J. P., and Mauch-Mani, B. 2005. Dissecting the $\beta$-aminobutyric acid-induced priming phenomenon in Arabidopsis. Plant Cell 17:987-999.

Ton, J., and Mauch-Mani, B. 2004. $\beta$-amino-butyric acid-induced resistance against necrotrophic pathogens is based on ABA-dependent priming for callose. Plant J. 38:119-130.

Vaahtera, L., and Brosché, M. 2011. More than the sum of its partsHow to achieve a specific transcriptional response to abiotic stress. Plant Sci. 180:421-430.

Vailleau, F., Daniel, X., Tronchet, M., Montillet, J. L., Triantaphylidès, C., and Roby, D. 2002. A R2R3-MYB gene, AtMYB30, acts as a positive regulator of the hypersensitive cell death program in plants in response to pathogen attack. Proc. Natl. Acad. Sci. U.S.A. 99:10179-10184.

van Hulten, M., Pelser, M., van Loon, L. C., Pieterse, C. M., and Ton, J. 2006. Costs and benefits of priming for defense in Arabidopsis. Proc. Natl. Acad. Sci. U.S.A. 103:5602-5607.

Van Kan, J. A. L., Stassen, J. H. M., Mosbach, A., Van Der Lee, T. A. J., Faino, L., Farmer, A. D., Papasotiriou, D. G., Zhou, S., Seidl, M. F., Cottam, E., Edel, D., Hahn, M., Schwartz, D. C., Dietrich, R. A., Widdison, S., and Scalliet, G. 2017. A gapless genome sequence of the fungus Botrytis cinerea. Mol. Plant Pathol. 18:75-89.

Vitrac, X., Bornet, A., Vanderlinde, R., Valls, J., Richard, T., Delaunay, J. C., Mérillon, J. M., and Teissédre, P. L. 2005. Determination of stilbenes ( $\varepsilon$-viniferin, trans-astringin, trans-piceid, cis- and trans-resveratrol, $\varepsilon$-viniferin) in Brazilian wines. J. Agric. Food Chem. 53:5664-5669.

Wang, K., Li, C., Lei, C., Jiang, Y., Qiu, L., Zou, X., and Zheng, Y. 2020. $\beta$-aminobutyric acid induces priming defence against Botrytis cinerea in grapefruit by reducing intercellular redox status that modifies posttranslation of $V v N P R 1$ and its interaction with $V v T G A 1$. Plant Physiol. Biochem. 156:552-565.
Wang, K., Li, C., Lei, C., Zou, Y., Li, Y., Zheng, Y., and Fang, Y. 2021. Dual function of $V v W R K Y 18$ transcription factor in the $\beta$-aminobutyric acid-activated priming defense in grapes. Physiol. Plant. 172:14771492

Wang, K., Liao, Y., Xiong, Q., Kan, J., Cao, S., and Zheng, Y. 2016. Induction of direct or priming resistance against Botrytis cinerea in strawberries by $\beta$-aminobutyric acid and their effects on sucrose metabolism. J. Agric. Food Chem. 64:5855-5865.

Wang, K. T., Liao, Y. X., Cao, S. F., Di, H. T., and Zheng, Y. H. 2015. Effects of benzothiadiazole on disease resistance and soluble sugar accumulation in grape berries and its possible cellular mechanisms involved. Postharvest Biol. Technol. 102:51-60.

Wang, K. T., Wu, D. Z., Bo, Z. Y., Chen, S., Wang, Z. R., Zheng, Y. H., and Fang, Y. 2019. Regulation of redox status contributes to priming defense against Botrytis cinerea in grape berries treated with $\beta$-aminobutyric acid. Sci. Hortic. (Amsterdam) 244:352-364.

Wilkinson, S. W., Pastor, V., Paplauskas, S., Pétriacq, P., and Luna, E. 2018. Long-lasting $\beta$-aminobutyric acid-induced resistance protects tomato fruit against Botrytis cinerea. Plant Pathol. 67:30-41.

Wilson, A. M., Work, T. M., Bushway, A. A., and Bushway, R. J. 1981. HPLC determination of fructose, glucose, and sucrose in potatoes. J. Food Sci. 46:300-301.

Xiao, S., and Chye, M. L. 2011. Overexpression of Arabidopsis ACBP3 enhances NPR1-dependent plant resistance to Pseudomonas syringe pv tomato DC3000. Plant Physiol. 156:2069-2081.

Zavaliev, R., Mohan, R., Chen, T., and Dong, X. 2020. Formation of NPR1 condensates promotes cell survival during the plant immune response. Cell 182:1093-1108.e18.

Zhai, R., Wang, Z., Zhang, S., Meng, G., Song, L., Wang, Z., Li, P., Ma F., and Xu, L. 2016. Two MYB transcription factors regulate flavonoid biosynthesis in pear fruit (Pyrus bretschneideri Rehd.). J. Exp. Bot. 67:1275-1284

Zhang, Z., Qin, G., Li, B., and Tian, S. 2014. Knocking out Bcsas1 in Botrytis cinerea impacts growth, development, and secretion of extracellular proteins, which decreases virulence. Mol. Plant-Microbe Interact. 27:590-600

Zhang, Z. B., Zhu, J., Gao, J. F., Wang, C., Li, H., Li, H., Zhang, H. Q., Zhang, S., Wang, D. M., Wang, Q. X., Huang, H., Xia, H. J., and Yang, Z. N. 2007. Transcription factor AtMYB103 is required for anther development by regulating tapetum development, callose dissolution and exine formation in Arabidopsis. Plant J. 52:528-538.

Zhong, R., Richardson, E. A., and Ye, Z. H. 2007. The MYB46 transcription factor is a direct target of SND1 and regulates secondary wall biosynthesis in Arabidopsis. Plant Cell 19:2776-2792.

Zhou, J., Lee, C., Zhong, R., and Ye, Z. H. 2009. MYB58 and MYB63 are transcriptional activators of the lignin biosynthetic pathway during secondary cell wall formation in Arabidopsis. Plant Cell 21:248-266

Zimmerli, L., Jakab, G., Métraux, J. P., and Mauch-Mani, B. 2000 Potentiation of pathogen-specific defense mechanisms in Arabidopsis by $\beta$-aminobutyric acid. Proc. Natl. Acad. Sci. U.S.A. 97:1292012925.

Zimmerli, L., Métraux, J. P., and Mauch-Mani, B. 2001. ß-Aminobutyric acid-induced protection of Arabidopsis against the necrotrophic fungus Botrytis cinerea. Plant Physiol. 126:517-523.

Zou, B., Jia, Z., Tian, S., Wang, X., Gou, Z., L, B., and Dong, H. 2013. AtMYB44 positively modulates disease resistance to Pseudomonas syringae through the salicylic acid signalling pathway in Arabidopsis. Funct. Plant Biol. 40:304-313. 